\title{
Lung-resident eosinophils represent a distinct regulatory eosinophil subset
}

\author{
Claire Mesnil, ${ }^{1,2}$ Stéfanie Raulier, ${ }^{1}$ Geneviève Paulissen, ${ }^{1}$ Xue Xiao, ${ }^{2,3}$ Mark A. Birrell, ${ }^{4}$ Dimitri Pirottin,, ${ }^{1,2}$ Thibaut Janss, ${ }^{1}$ \\ Philipp Starkl, ${ }^{5}$ Eve Ramery, ${ }^{2}$ Monique Henket, ${ }^{6,7}$ Florence N. Schleich, ${ }^{6,7}$ Marc Radermecker, ${ }^{8}$ Kris Thielemans, ${ }^{9}$ Laurent Gillet, ${ }^{2,3}$ \\ Marc Thiry, ${ }^{10}$ Maria C. Belvisi, ${ }^{4}$ Renaud Louis, ${ }^{6,7}$ Christophe Desmet, ${ }^{1,2}$ Thomas Marichal, ${ }^{1,2}$ and Fabrice Bureau ${ }^{1,2,11}$ \\ 'Laboratory of Cellular and Molecular Immunology, Groupe Interdisciplinaire de Génoprotéomique Appliquée (GIGA) Research, ${ }^{2}$ Faculty of Veterinary Medicine, and ${ }^{3}$ Immunology-Vaccinology, Fundamental \\ and Applied Research for Animals and Health, University of Liège, Liège, Belgium. ${ }^{4}$ Respiratory Pharmacology, National Heart and Lung Institute, Faculty of Medicine, Imperial College London, London, \\ United Kingdom. ${ }^{5}$ CeMM Research Center for Molecular Medicine of the Austrian Academy of Sciences, and Department of Medicine I, Medical University of Vienna, Vienna, Austria. ${ }^{6}$ Department of \\ Pulmonary Medicine, 'aboratory of Pneumology, GIGA Research, and ${ }^{8}$ Department of Cardiovascular and Thoracic Surgery and Human Anatomy, Centre Hospitalier Universitaire (CHU), University of Liège, \\ Liège, Belgium. 'aboratory of Molecular and Cellular Therapy, Vrije Universiteit Brussel, Brussels, Belgium. ${ }^{10}$ Laboratory of Cellular and Tissular Biology, GIGA Research, University of Liège, Liège, Belgium. \\ ${ }^{11}$ Walloon Excellence in Lifesciences and Biotechnology (WELBIO), Wallonia, Belgium.
}

\begin{abstract}
Increases in eosinophil numbers are associated with infection and allergic diseases, including asthma, but there is also evidence that eosinophils contribute to homeostatic immune processes. In mice, the normal lung contains resident eosinophils (rEos), but their function has not been characterized. Here, we have reported that steady-state pulmonary rEos are IL-5-independent parenchymal Siglec-Fint $C D 62 L^{+} C D 101^{10}$ cells with a ring-shaped nucleus. During house dust mite-induced airway allergy, rEos features remained unchanged, and rEos were accompanied by recruited inflammatory eosinophils (iEos), which were defined as IL-5-dependent peribronchial Siglec- ${ }^{\text {hi }}$ CD62L-CD101 ${ }^{\text {hi }}$ cells with a segmented nucleus. Gene expression analyses revealed a more regulatory profile for rEos than for iEos, and correspondingly, mice lacking lung rEos showed an increase in Th2 cell responses to inhaled allergens. Such elevation of Th2 responses was linked to the ability of rEos, but not iEos, to inhibit the maturation, and therefore the pro-Th2 function, of allergen-loaded DCs. Finally, we determined that the parenchymal rEos found in nonasthmatic human lungs (Siglec- $8^{+}$CD62L + IL-3R ${ }^{10}$ cells) were phenotypically distinct from the iEos isolated from the sputa of eosinophilic asthmatic patients (Siglec- $8+$ CD62 $L^{10} I L-3 R^{\text {hi }}$ cells), suggesting that our findings in mice are relevant to humans. In conclusion, our data define lung rEos as a distinct eosinophil subset with key homeostatic functions.
\end{abstract}

\section{Introduction}

Eosinophils are terminally differentiated granulocytes that develop in the BM under the control of key transcription factors, mainly GATA1, C/EBP $\alpha$, PU.1, and XBP1 (1-6). They are characterized by the presence in their cytoplasm of specific secondary granules containing toxic cationic proteins, which are stainable with acid aniline dyes and Congo red $(1,7)$. Eosinophil granules contain a crystalloid core made of major basic proteins 1 and 2 (MBP1 and MBP2) and a matrix composed of eosinophil cationic protein, eosinophil peroxidase (EPO), and eosinophil-derived neurotoxin (1).

Under physiological conditions, only small numbers of eosinophils are released from the BM. By contrast, eosinophilopoiesis is dramatically increased as a result of Th2 cell responses associated with helminth infections or allergic diseases such as asthma $(1,2,8)$. This increase in eosinophil production is driven by a dedicated set

\section{Related Commentary: p. 3185}

Authorship note: C. Mesnil and S. Raulier contributed equally to this work and are co-first authors. T. Marichal and F. Bureau jointly directed this work and are co-last authors. Conflict of interest: The authors have declared that no conflict of interest exists. Submitted: November 30, 2015; Accepted: June 9, 2016.

Reference information: / Clin Invest. 2016;126(9):3279-3295. doi:10.1172/JCI85664. of cytokines, namely IL-3, IL-5, and granulocyte macrophage-CSF (GM-CSF) $(1,8)$. Among these, the Th2-associated cytokine IL-5 is the most specific cytokine for the eosinophil lineage and is responsible not only for expansion of eosinophils from committed BM progenitors, but also for their release into the blood and their survival following migration into the tissues $(1,8,9)$. Once at the site of injury, eosinophils can release their cytotoxic granule proteins as well as preformed cytokines and lipid mediators, contributing in certain circumstances to parasite destruction, but also to exacerbation of inflammation and tissue damage, which is particularly deleterious when Th2 responses are directed against allergens (1, $8,10)$. It is interesting to note that the role of eosinophils is not limited to the effector phase of Th2 responses but also seems to be important for the induction of adaptive immunity. Indeed, there is evidence of antigen presentation by eosinophils in some experimental models of Th2 responses against helminths or allergens (11-13), and recent studies have further shown that eosinophils can also promote Th2 immunity through EPO-mediated induction of DC activation and migration $(14,15)$.

Accumulating evidence indicates that, besides their roles in Th2 responses, eosinophils also regulate homeostatic processes at steady state, thereby challenging the exclusive paradigm of the eosinophil as a destructive and inflammatory cell $(1,2,8,16)$. 
In fact, under baseline conditions, eosinophils rapidly leave the bloodstream to enter tissues, mainly the gastrointestinal tract, adipose tissue, thymus, uterus, and mammary glands, where they regulate a variety of important biological functions $(1,2,8,16)$. The vast majority of these tissue-resident eosinophils (rEos) are found in the nonesophageal portions of the gastrointestinal tract, where they promote IgA class switching and the maintenance of IgA-expressing plasma cells $(17,18)$. They also support the development of Peyer's patches and mucus production in the small intestine (18). rEos present in the adipose tissue produce IL-4, thereby favoring the polarization of adipose macrophages toward the alternatively activated phenotype (19). Alternatively activated macrophages play a crucial role in glucose homeostasis and development of beige fat $(19,20)$, which is known to protect against obesity (21). In the thymus, rEos have the capacity to promote thymocyte apoptosis and are therefore thought to contribute to the process of negative $\mathrm{T}$ cell selection (22). Finally, a role for $\mathrm{rEos}$ in preparing the uterus for pregnancy and in regulating mammary gland development has been proposed $(23,24)$.

While rEos have also been identified in the mouse lung (25-27), they have not been fully characterized, and their role remains to be determined. Here, we show that mouse lung rEos display unique morphological and phenotypical features that unambiguously distinguish them from the inflammatory eosinophils (iEos) that are recruited to the lung during house dust mite (HDM)-induced allergic airway inflammation. Moreover, we demonstrate that lung rEos are endowed with the striking capacity to prevent the development of pulmonary Th2 immunity against inhaled allergens, thereby contributing to the maintenance of lung homeostasis in mice. Finally, by comparing lung rEos and iEos in humans, we provide evidence that our findings in mice are relevant to humans.

\section{Results}

Lung $r$ Eos are parenchymal Siglec- $F^{\text {int }} C D 125^{\text {int }}$ cells with a ring-shaped nucleus. First, we aimed to better characterize lung rEos of naive mice. In order to avoid any contamination by blood cells, whole lungs of adult C57BL/6 mice were first extensively flushed with PBS through the right ventricle and were then digested to obtain a single-cell suspension. Using a flow cytometry-based approach, lung eosinophils were delineated, among living singlet CD $45.2^{+}$ cells (Supplemental Figure 1; supplemental material available online with this article; doi:10.1172/JCI85664DS1), with a combination of Abs recognizing Siglec-F and the subunit $\alpha$ of the IL-5 receptor (IL-5R $\alpha$ or CD125) (28-31). This approach identified 3 cell populations, namely intermediate Siglec-F (Siglec- $F^{\text {int }}$ ) CD125 $5^{\text {int }}$, Siglec-F ${ }^{\text {hi }} \mathrm{CD} 125^{\text {int }}$, and Siglec- $\mathrm{F}^{\mathrm{dim}} \mathrm{CD} 125^{\text {hi }}$ cells (Figure 1A). The Siglec-F ${ }^{\text {int }}$ CD125 ${ }^{\text {int }}$ cell population exhibited features of eosinophils, i.e., an eosinophilic cytoplasm (Figure 1B), expression of F4/80 (32), and expression of the eosinophil eotaxin receptor (CCR3) (33) (Figure 1C). Notably, Siglec- $F^{\text {int }}$ CD125 $5^{\text {int }}$ eosinophils consistently exhibited a ring-shaped nucleus (Figure 1B). Siglec- $F^{\text {hi }}$ CD $125^{\text {int }}$ and Siglec-F ${ }^{\text {dim }} C D 125^{\text {hi }}$ cells were identified as F4/80 ${ }^{\text {hi CCR3 }}{ }^{-}$alveolar macrophages (AMs) and F4/80-CCR3neutrophils, respectively (Figure 1, B and C). Ultrastructural analysis of FACS-sorted Siglec- $F^{\text {int }}$ CD $125^{\text {int }}$ rEos revealed secondary granules with variable levels of density (Figure 1D, high [arrows], intermediate [asterisks], and low [arrowheads] density, and Sup- plemental Figure 2), suggesting that they were undergoing piecemeal degranulation, a process during which granule proteins are released gradually $(34,35)$. Some low-density granules displayed a central structure that most likely corresponded to the central MBP-containing core typical of eosinophil granules $(34,35)$, and contained intragranular vesiculotubular structures previously delineated within human eosinophilic granules (35) (Supplemental Figure 2, arrows and arrowheads, respectively).

In an attempt to determine whether rEos had phagocytic properties, naive mice were instilled intratracheally (i.t.) with fluorescent beads, and fluorescence was assessed 6 hours later in lung rEos, AMs, and T lymphocytes (used as positive and negative controls, respectively) by flow cytometry and confocal microscopy (Supplemental Figure 3A). rEos were able to uptake beads, as an average of $8.7 \% \pm 2.3 \%$ (mean \pm SEM) of these rEos showed fluorescence, which was associated with the physical presence of beads in their cytoplasm (Figure 1E and Supplemental Figure 3). Such experiment also supported the fact that rEos were truly located within the lung tissue and were not associated with the lung vessels. Indeed, beads administered locally via the airways were taken up by lung rEos but not by blood monocytes or blood eosinophils (Supplemental Figure 3B). Assessment of the localization of more than 100 lung eosinophils on Congo red- and MBP-stained lung tissue sections from naive mice further confirmed that the vast majority of the eosinophils were parenchymal (Figure 1, F and G), with less than $10 \%$ of eosinophils found in the vicinity of bronchi or inside the blood vessels.

rEos were not present at birth but rapidly accumulated in the lung until day 7, and the numbers of rEos per milligram of lung tissue did not change significantly from days 7 to 42 after birth (Figure $1 \mathrm{H})$. In adult mice, rEos were found in comparable numbers in the lungs of male and female mice (Figure 1I) and were completely absent from the broncho-alveolar lavage fluid (BALF) (data not shown). In mice from the BALB/c background, lung rEos were

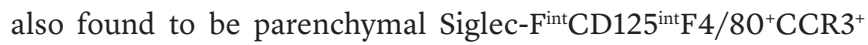
cells with a ring-shaped nucleus (Supplemental Figure 4).

Lung rEos are not affected by allergic inflammation and differ from iEos. In the lung, eosinophils are well described in the context of allergic asthma, where they can release granule-associated cytotoxic molecules and contribute to the inflammatory response $(36,37)$. In order to determine how $r E o s$ are influenced by allergic inflammation, we assessed the numbers, phenotype, and localization of rEos in the lungs of allergic C57BL/6 mice during the effector phase of HDM-induced airway allergy (38) (Figure 2A). Figure 2B shows that the population of lung rEos observed in saline-injected control mice (rEos at steady state, referred to hereafter as $\mathrm{rEos}_{\mathrm{ss}}$ ) was present in similar numbers in HDM-treated mice (rEos in inflammatory conditions, referred to hereafter as $\left.\mathrm{rEos}_{\mathrm{i}}\right)$. Moreover, $\mathrm{rEos}_{\mathrm{i}}$ had a similar ring-shaped nucleus and expressed the same levels of Siglec-F, CD125, $\mathrm{F} 4 / 80$, and CCR3 as did $\mathrm{rEos}_{\mathrm{ss}}$ (blue color, Figure 2, C-E). In addition, we found that the numbers of parenchymal eosinophils remained unchanged after the onset of airway allergy (Figure 2, G-I), supporting the idea that these cells probably corresponded to the $\mathrm{EDos}_{\mathrm{i}}$ detected by flow cytometry (Figure 2, B and C).

As expected in this model of HDM-induced asthma, a population of iEos was recruited into the lungs. iEos showed higher 


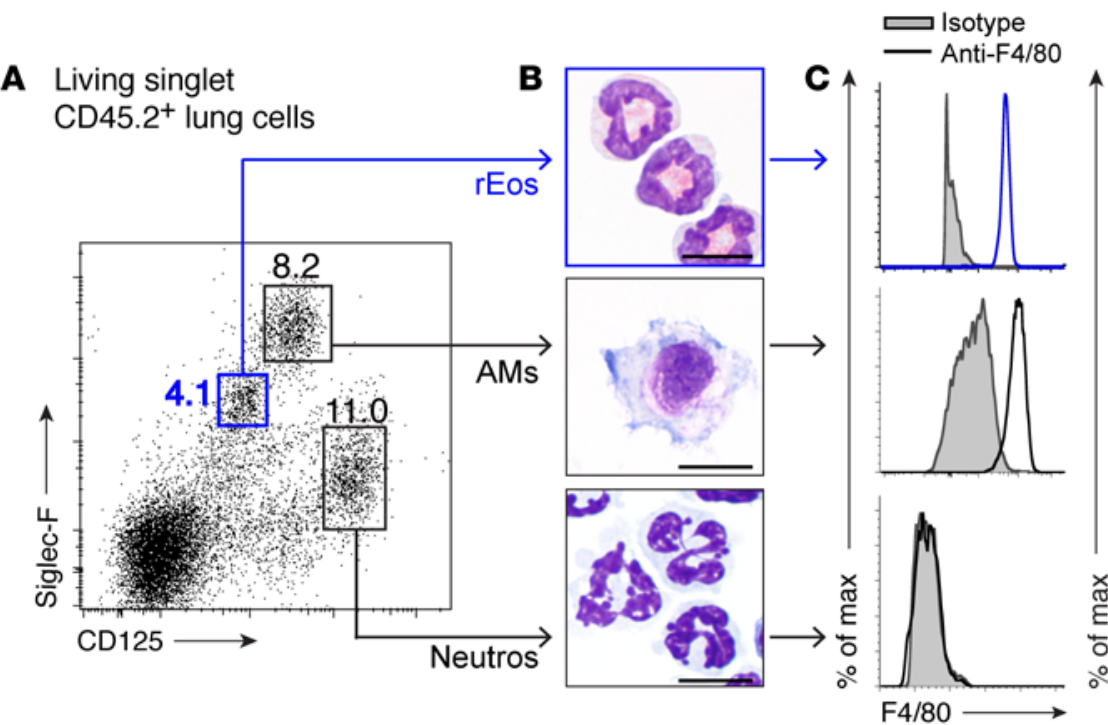

D

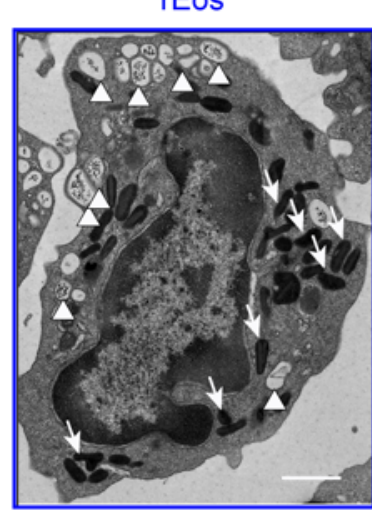

rEos-associated granules

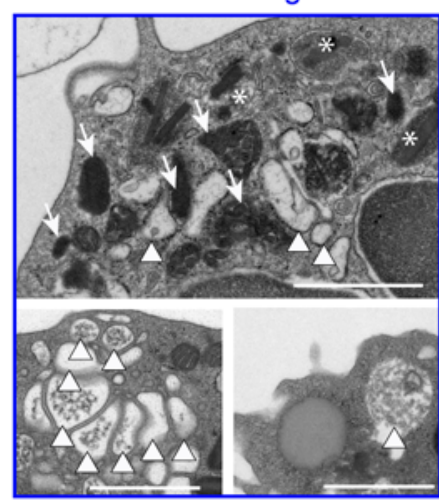

E

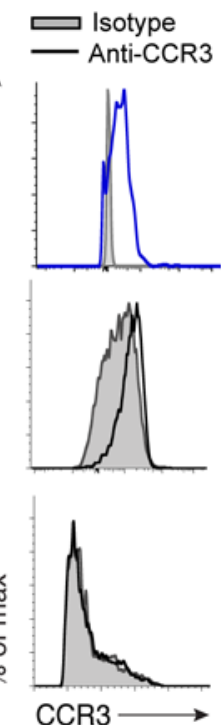

rEos

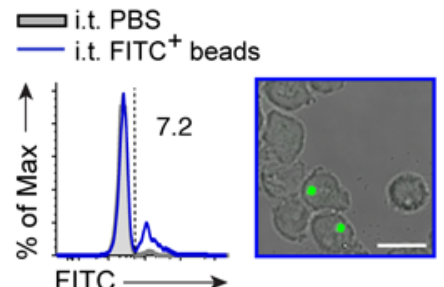

G

MBP
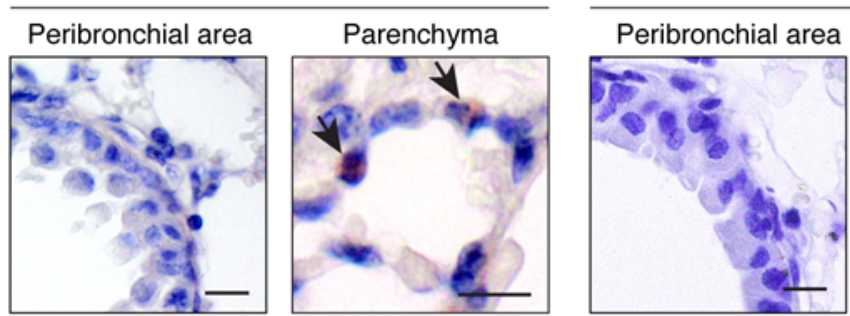

Parenchyma

H

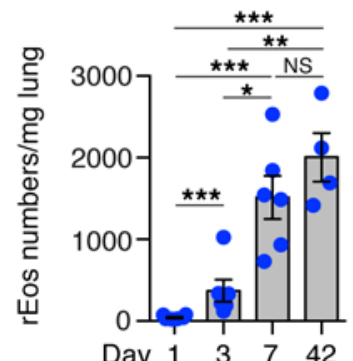

I

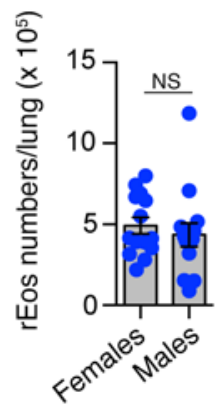

Figure 1. Characterization of lung rEos in naive C57BL/6 mice. (A) Dot plot of lung leukocytes according to Siglec-F and CD125 expression. Numbers indicate the percentage of gated cells. Neutros, neutrophils. (B) Photographs of the FACS-sorted cell populations gated in A. (C) Histograms of F4/80 and CCR3 expression in the cell populations shown in B. (D) Representative electron microscopy photographs of rEos (left panel, representative of more than 100 individual cells analyzed) and rEos-associated granules (right panels). Arrows, asterisks, and arrowheads indicate secondary granules with high, intermediate, and low densities, respectively. (E) Phagocytic potential of rEos 6 hours after local i.t. instillation of fluorescent beads. Number indicates the percentage of bead-positive cells. (F) Congo red- and (G) MBP-stained lung sections. Arrows indicate Congo red-and MBP-positive eosinophils, respectively. (H) Kinetics of appearance of lung rEos after birth. (I) Absolute numbers of rEos in the lungs of female and male mice. ( $H$ and $\mathbf{I})$ Results represent the mean \pm SEM as well as individual values. ${ }^{*} P<0.05$, ${ }^{* *} P<0.01$, and ${ }^{* *} P<0.001$, by 1-way ANOVA, followed by Tukey's test for multiple comparisons on log-transformed values $(\mathbf{H})$ or by 2-tailed Student's $t$ test on square root-transformed values (I). Data in $\mathbf{A}-\mathbf{G}$ are representative of 1 of more than 8 mice, each giving similar results. Data in $\mathbf{H}$ and I were pooled from 2 and 3 independent experiments, respectively ( $n=4-6$ / group and 14/group, respectively). Scale bars: $1 \mu \mathrm{m}$ (D) and $10 \mu \mathrm{m}$ (B, E-C). Max, maximum. 
levels of Siglec-F expression (red color, Figure 2C), a highly segmented nucleus (Figure 2D), and were also positive for F4/80 and CCR3 (Figure 2E). Electron microscopic analysis of FACS-sorted iEos revealed dense secondary granules, without apparent signs of degranulation (Figure 2F, arrows; representative of $>100$ cells analyzed) and a segmented nucleus (Figure $2 F)$. HDM-induced iEos, unlike rEos, were mainly localized in the peribronchial area (Figure 2, G-I) and were recruited in the BALF (Figure 2, J and K). Similar results were found in BALB/c mice (Supplemental Figure 5).

Lung rEos and iEos have distinct gene expression profiles. So far, our data suggest that rEos are not influenced by the HDM-induced allergic environment and are distinct from iEos. To further confirm these observations, we isolated steady-state rEos from naive mice $\left(\mathrm{rEos}_{\mathrm{ss}}\right)$, rEos from HDM-treated mice ( $\mathrm{rEos}_{\mathrm{i}}$ ), and iEos from HDM-treated mice (iEos) (Supplemental Figure 6) and compared their transcriptome by microarrays. Hierarchical clustering based on all expressed genes showed that $\mathrm{rEos}_{\mathrm{ss}}$ clustered together with rEos $_{i}$ (Supplemental Figure 7A). Conversely, the transcriptomic signature of iEos was distant from that of rEos, regardless of whether they originated from naive or HDM-treated allergic mice. Indeed, the coefficients of correlation were very low between iEos and $r E o s(r<0.25$, Supplemental Figure 7A). Volcano plot representation of the differentially expressed genes between $\mathrm{rEos}_{\mathrm{ss}}$ and $\mathrm{rEos}_{\mathrm{i}}$ showed that, using a biological fold change (FC) of 2 and a $P$ value of less than 0.01 , not a single gene was differentially expressed between $\mathrm{rEos}_{\mathrm{ss}}$ and $\mathrm{rEos}_{\mathrm{i}}$ (Figure 3A). Increasing the threshold to a $P$ value of less than 0.05 only revealed 3 differentially expressed genes between these 2 cell populations (data not shown), strongly suggesting that rEos are not impacted by the HDM-induced proallergic environment. However, using the same thresholds ( $\mathrm{FC}>2 ; P<0.01)$, more than 160 genes were differentially expressed between iEos and $\mathrm{rEos}_{\mathrm{ss}}$ and between iEos and $\mathrm{rEos}_{\mathrm{i}}$ (Figure 3A and Supplemental Figure 7B), reinforcing the idea that iEos and rEos represent distinct cell populations.

On the basis of a thorough bibliographical search, we selected the differentially expressed genes known to be associated with the modulation of inflammatory and immune responses, which led to 28 genes whose expression was upregulated in iEos and 20 genes whose expression was upregulated in rEos (Figure 3B). iEos had high expression of several proinflammatory genes such as Slc3a2 (39), Tlr4 (40), C3ar1 (41, 42), Il13ra1 (43), and Il6 (44), consistent with the known function of iEos as proinflammatory effector cells (Figure 3B). In contrast, rEos expressed several genes such as Anxa1 (45, 46), Nedd4 (47), Runx3 (48), Serpinb1a (49), and Ldlr (50) that are implicated in the negative regulation of immune responses and tissue homeostasis (Figure 3B). These analyses suggested that, in addition to their unique localization and morphology, rEos and iEos were also functionally different.

Expression of CD62L and CD101 discriminates between mouse lung $r$ Eos and $i$ Eos. Given the list of differentially expressed genes shown in Figure 3B, we sought to find surface markers allowing a better discrimination between rEos and iEos. Expression of several surface molecules was assessed by flow cytometry on $\mathrm{rEos}_{\mathrm{ss}}, \mathrm{rEos}_{\mathrm{i}}$, and iEos. We found that L-selectin, also known as CD62L (encoded by Sell in mice, see Figure 3B), was specifically expressed on the surface of $\mathrm{rEos}_{\mathrm{ss}}$ and $\mathrm{rEos}_{\mathrm{i}}$, albeit at variable levels, but not on the surface of iEos (Figure 4, A-C). Conversely, $\mathrm{rEos}_{\mathrm{ss}}$ and $\mathrm{rEos}_{\mathrm{i}}$ expressed low levels of CD101, whereas CD101 was highly expressed on the surface of iEos (Figure 4, A-C). Notably, CD101 could be used to distinguish between $\mathrm{rEos}_{\mathrm{i}}\left(\mathrm{CD} 101^{\mathrm{lo}}\right)$ and $i E o s\left(C D 101^{\text {hi }}\right)$ when gating on the total population of eosinophils in the lungs of HDM-challenged mice (Figure 4D). Interestingly, the expression levels of CD62L and CD101 on rEos ${ }_{s s}$ were similar to those seen on $\mathrm{rEos}_{1}$ from HDM-treated allergic mice (Figure 4, A and B), concordant with our microarray data (Figure $3 \mathrm{~B})$ and with the hypothesis that rEos, like iEos, are differentiated cells released from the BM that are not able to modify their phenotype over the course of allergic tissue inflammation. If this were true, the 2 Siglec- $\mathrm{F}^{\text {int }} \mathrm{CD} 62 \mathrm{~L}^{+} \mathrm{CD} 101^{\text {lo }}$ (i.e., $\mathrm{rEos}_{\mathrm{i}}$ ) and Siglec$\mathrm{F}^{\text {hi }} \mathrm{CD} 62 \mathrm{~L}^{-} \mathrm{CD} 101^{\text {hi }}$ (i.e., iEos) eosinophil populations present in the lungs should also be detected in the blood. We verified this assumption and found that only Siglec- $\mathrm{F}^{\mathrm{int}} \mathrm{CD} 62 \mathrm{~L}^{\text {hi }} \mathrm{CD} 101^{-}$rEoslike cells were present in the blood of naive mice (Figure 5A). However, 2 phenotypically distinct populations of rEos- and iEos-like (Siglec- $\mathrm{F}^{\mathrm{hi}} \mathrm{CD} 62 \mathrm{~L}^{\mathrm{lo}} \mathrm{CD} 101^{+}$) cells were indeed found in the blood of HDM-treated allergic mice (Figure 5B), suggesting that both lung rEos and iEos are terminally differentiated cells.

Given that small intestinal eosinophils (Int Eos) have previously been shown to be phenotypically different from lung rEos (26), we extended this analysis by comparing their expression of CD62L and CD101. Int Eos were found to be Siglec- ${ }^{\text {int }} C D 125^{\text {int }} C D 101^{-}$cells expressing F4/80 and CCR3 and exhibited a ring-shaped nucleus. However, as compared with lung rEos, which expressed CD62L, Int Eos were found to be negative for CD62L (Supplemental Figure 8).

rEos are independent of IL-5 for their presence in the lung. IL-5 is important for the expansion of eosinophils in the BM, eosinophil release, and survival in the blood in response to different inflammatory stimuli $(1,8,9,51)$. Yet, basal blood eosinophil numbers remain unchanged in $I l 5^{-/-}$mice (52), and, as shown in Figure 2B, the numbers of lung rEos remained unaffected by the presence of allergic inflammation, at a time point when IL-5 levels were significantly increased in the lungs (Supplemental Figure 9, A and B). Hence, we next wondered to what extent rEos and iEos were dependent on IL-5 for their presence in the blood and for their recruitment to the lung tissue. First, we treated naive C57BL/6 mice i.p. with $100 \mu \mathrm{g}$ neutralizing Abs directed against IL-5 ( $\alpha-$ IL-5) and quantified the numbers of rEos in the lungs 24 hours later (Figure 6A, left panel). $\alpha$-IL-5 treatment did not significantly affect the numbers of $\mathrm{rEos}_{\mathrm{ss}}$ in the lungs of naive animals (Figure 6B, left panel) or the numbers of rEos-like blood eosinophils (Figure 6C, left panel). In addition, the numbers of lung rEos remained unchanged in naive mice treated 3 times daily with 100 $\mu \mathrm{g} \alpha-\mathrm{IL}-5$, which corresponds to the time during which $90 \%$ of lung rEos are replaced (26) (Figure 6D). Second, HDM-sensitized and challenged C57BL/ 6 mice were treated i.p. with $100 \mu \mathrm{g} \alpha-\mathrm{IL}-5$ one hour before the last intranasal (i.n.) HDM challenge (Figure $6 \mathrm{~A}$, right panel), and the numbers of $\mathrm{rEos}_{\mathrm{i}}$ and iEos were evaluated 3 days later. Interestingly, $\alpha-\mathrm{IL}-5$ treatment did not impact the numbers of Eos $_{i}$ but substantially inhibited the recruitment of iEos in the lungs of $\alpha$-IL-5-treated mice compared with that seen in isotype-injected mice (Figure 6B, right panel). Similarly, rEoslike blood eosinophil levels seemed not to be affected by $\alpha-$ IL- 5 treatment, while iEos-like blood eosinophil levels were signifi- 
A

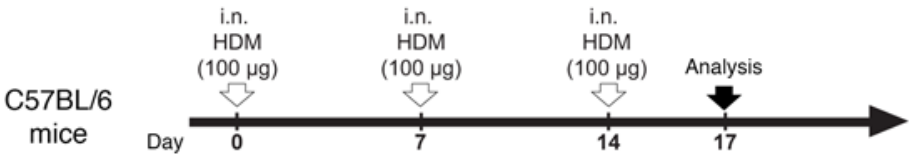

C Living singlet CD45.2+ lung cells from HDM-treated mice

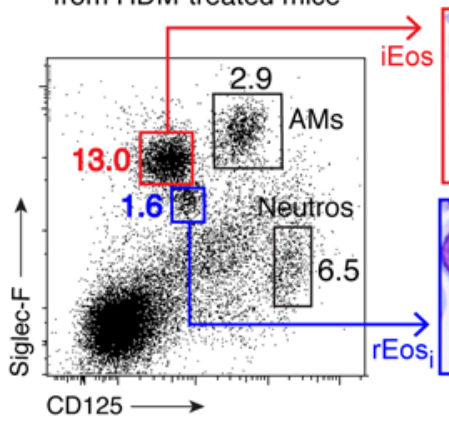

D

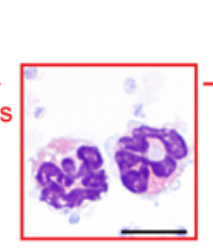

$\mathbf{E}$

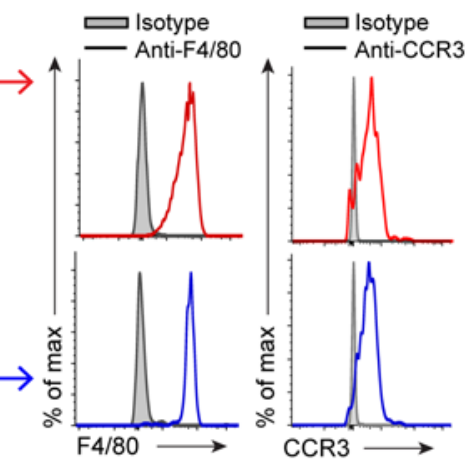

B
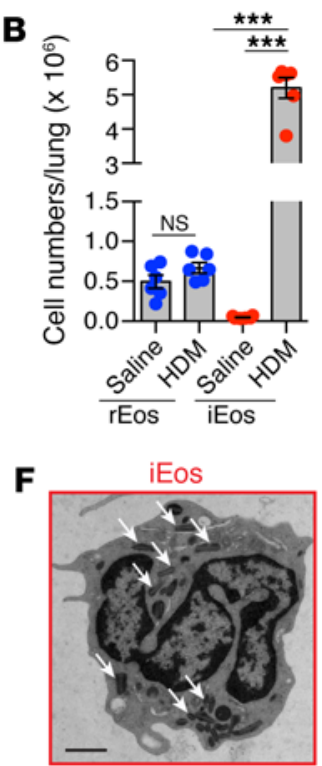

I

Congo red

Parenchymal Peribronchial

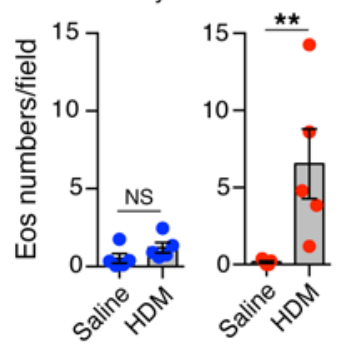

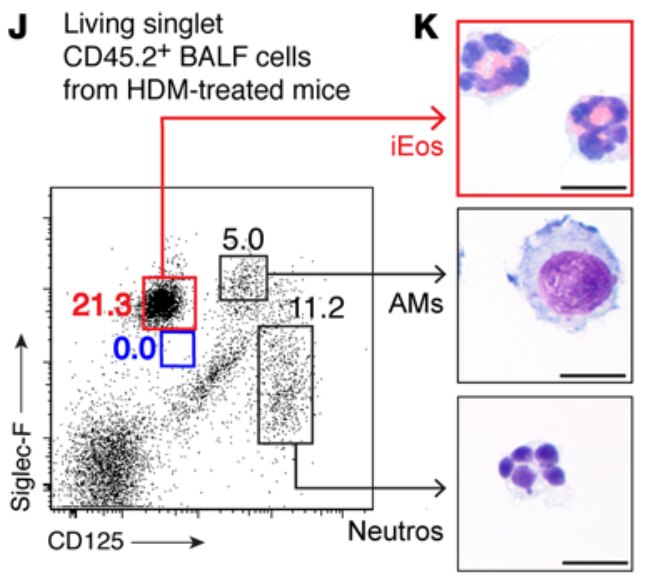

Figure 2. Characterization of lung rEos and iEos in HDM-challenged C57BL/6 mice. (A) Experimental outline. i.n., intranasal. (B) Absolute numbers of lung eosinophil subsets. (C) Dot plot of lung leukocytes according to Siglec-F and CD125 expression. Numbers indicate the percentage of gated cells. (D) Photographs of the 2 lung eosinophil subsets. (E) Histograms of F4/80 and CCR3 expression in the cell populations shown in $\mathbf{D}$. (F) Representative electron microscopy photograph of iEos (>100 individual cells analyzed). Arrows indicate secondary granules. (G) Congo red- and (H) MBP-stained lung sections. Arrows indicate Congo red- and MBP-positive eosinophils, respectively. (I) Quantification of parenchymal and peribronchial eosinophils in Congo red-stained lung sections. Eos, eosinophils. (J) Dot plot of BALF leukocytes according to Siglec-F and CD125 expression. Numbers indicate the percentage of gated cells. (K) Photographs of the gated cell populations in J. (B and I) Results represent the mean \pm SEM as well as individual values ( $n=5-6 /$ group) and are representative of 1 of more than 4 independent experiments. ${ }^{*} P<0.01$ and ${ }^{* *} P<0.001$, by (B) a mixed-effects model, followed by Tukey's test for multiple comparisons on log-transformed values, and (I) a Mann-Whitney $U$ test. (C-H) Data are representative of 1 of more than 8 mice, each of them giving similar results. Scale bars: $10 \mu \mathrm{m}(\mathbf{D}, \mathbf{G}$, and $\mathbf{H})$ and $1 \mu \mathrm{m}(\mathbf{F})$. Neutros, neutrophils.

cantly decreased (Figure 6C, right panel). In addition, injection of recombinant IL-5 (rIL-5) into naive mice did not promote the recruitment of rEos-like cells or rEos in the blood and the lungs, respectively (Supplemental Figure 9, C and D). These results support the idea that rEos, as opposed to iEos, do not depend on IL-5 for their presence in the blood or recruitment to the lungs.

Nevertheless, the fact that rEos and iEos expressed similar levels of IL-5R $\alpha$ (CD125) (see Figure 2C) suggested that rEos, like iEos, could respond to IL-5. To test this hypothesis, we assessed the activation of ERK1/2 and the survival of primary lung $r$ Eos and iEos stimulated in vitro with or without rIL-5. We found that rIL-5 treatment induced a significant increase in the levels of phosphorylated ERK (p-ERK) in $\mathrm{rEos}_{\mathrm{ss}}$ from naive mice, as well as in $\mathrm{rEos}_{\mathrm{i}}$ and iEos from HDM-treated allergic mice (Figure 6E). AMs were chosen as negative controls. In addition, rIL-5 significantly promoted survival of the 3 FACS-sorted eosinophil subpopulations, with an effect that was significantly more pronounced for rEos than for iEos 24 hours after treatment (Figure 6F). Altogether, our data obtained from in vivo experiments indicate that the presence of lung rEos and blood rEos-like eosinophils are independent of IL-5, even though $\mathrm{rEos}$ can respond to rIL-5 in vitro.

rEos, but not iEos, can inhibit the development of Th2 responses to HDM and the pro-Th2 potential of DCs. Our microarray data suggested that lung rEos could play immunoregulatory roles (Figure $3 \mathrm{~B})$. This is consistent with recent studies showing homeostatic functions for eosinophils in several organs $(8,16,53)$. These 
A

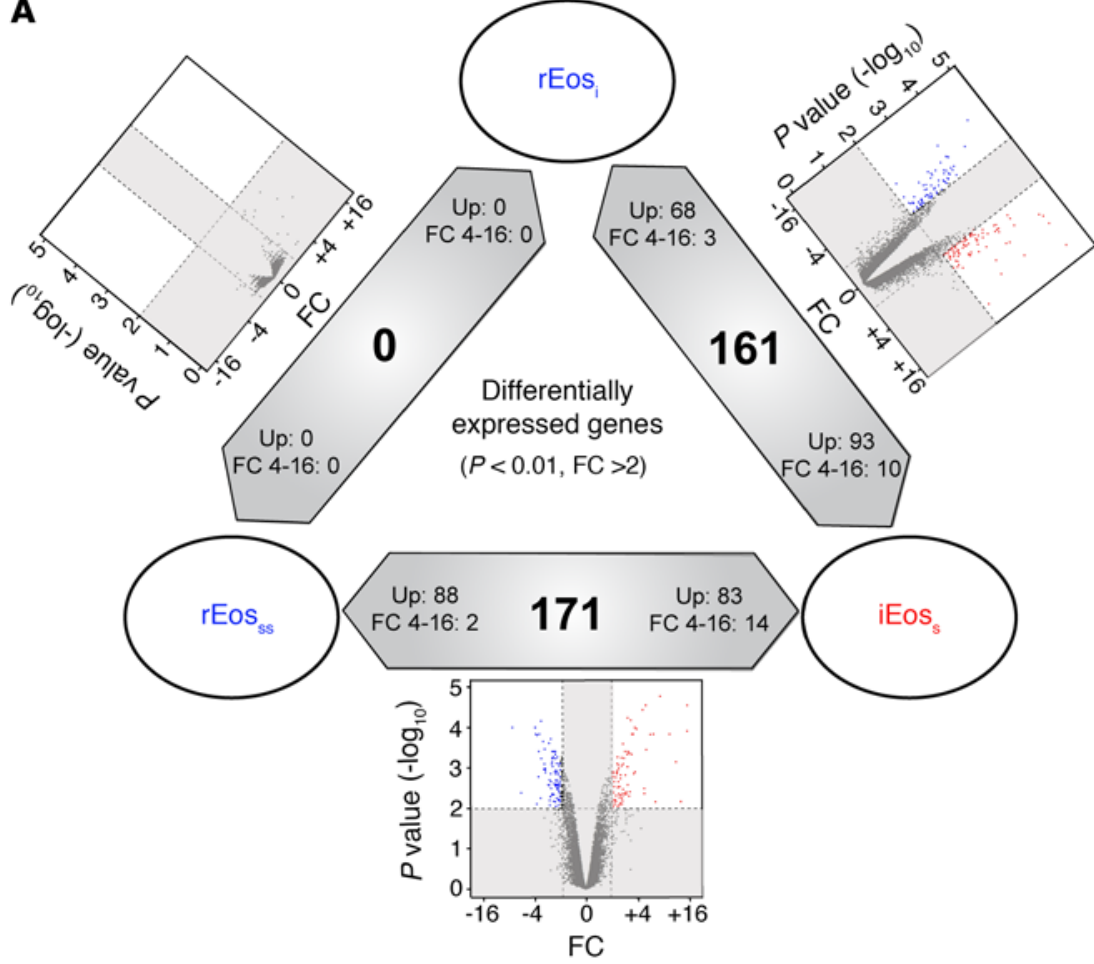

B

\begin{tabular}{|c|c|c|c|c|c|c|}
\hline \multicolumn{3}{|c|}{ iEos } & \multicolumn{3}{|c|}{$\mathrm{rEos}_{\mathrm{ss}}$} & \multirow[t]{2}{*}{ rEos } \\
\hline 1 & 2 & 3 & 1 & 2 & 3 & \\
\hline
\end{tabular}

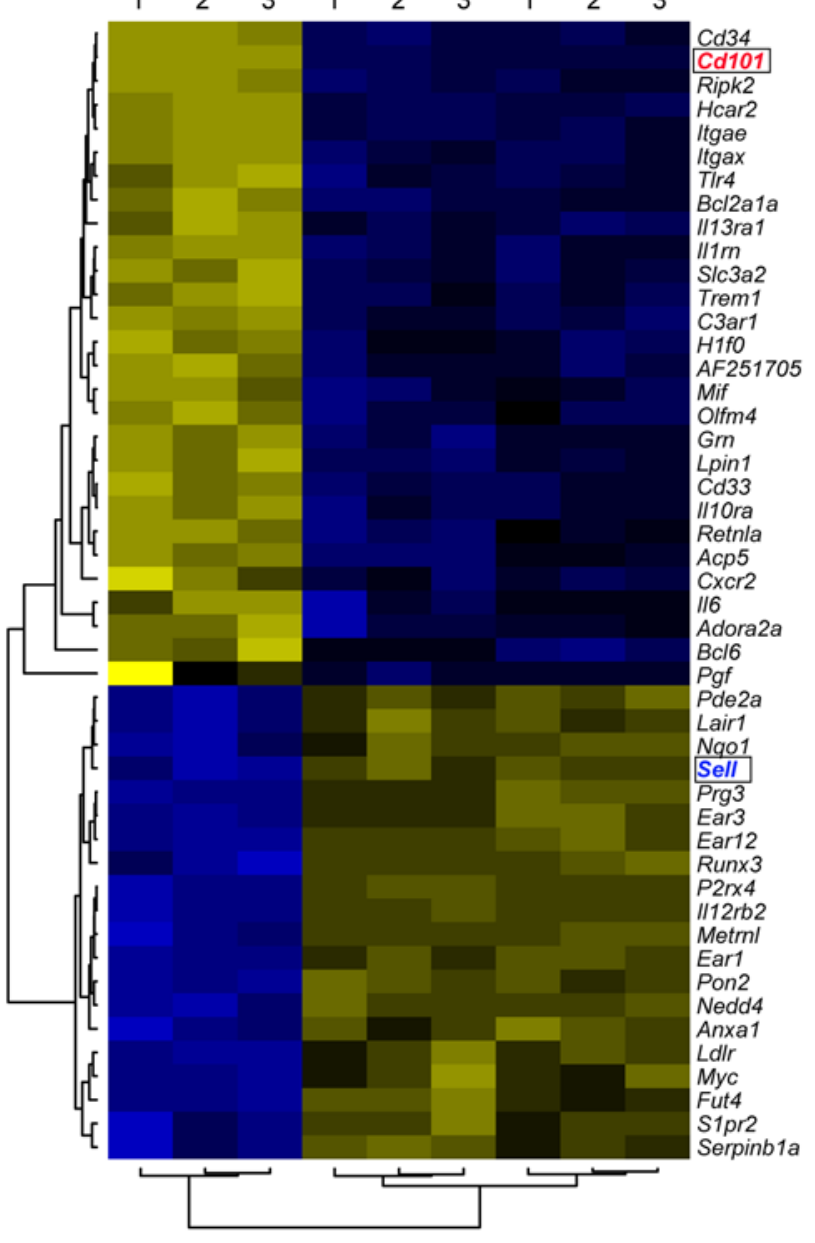

Figure 3. Transcriptomic profile of $\mathrm{rEos}_{\mathrm{ss}}$, rEos, and iEos. (A) Summary of differentially expressed genes $(P<0.01 ; \mathrm{FC}>2)$ in each pairwise comparison showing differentially expressed genes in red and blue in a volcano plot, the total number of differentially expressed genes in the middle of the bidirectional arrows, and arrowheads showing the direction of differential expression for all and highly (FC 4-16) differentially expressed genes. (B) Heatmap showing the relative expression of differentially expressed genes associated with the regulation of inflammatory and immune responses between iEos and rEos.

homeostatic effects have been demonstrated experimentally by the use of transgenic mice such as $\triangle$ dblGATA mice, in which a targeted deletion of the double palindromic GATA-1-binding site in the Gatal promoter results in eosinophil deficiency (54). Given that $\triangle$ dblGATA mice lacked lung rEos (Supplemental Figure 10A) and that only rEos, but not iEos, are present in the lungs under normal conditions, $\Delta \mathrm{dbl}$ GATA mice were chosen as a pertinent tool for studying rEos function.

In the gut, eosinophils have been shown to be required for gut homeostasis through the generation and maintenance of IgA $\mathrm{A}^{+}$plasma cells (17). However, the situation was different in the lungs, as the numbers of $\operatorname{IgA}^{+}$plasma cells were not reduced in the lungs of $\triangle$ dblGATA mice compared with numbers detected in the lungs of control mice (Supplemental Figure 10B).

A unique feature of the lung mucosa resides in its continuous exposure to allergens and immunostimulatory molecules present in the ambient air, and the lung innate immune system has developed strategies to ignore or tolerate these foreign antigens and prevent the development of aberrant Th2 responses that are classically associated with asthma (55-58). As rEos express genes, namely, Anxa1 (46), Runx3 (48), and Ldlr (50), involved in the prevention of Th2 and asthmatic responses (Figure 3B), we postulated that they could contribute to lung homeostasis by inhibiting airway sensitization to allergens. To test this hypothesis, rEos-deficient $\triangle$ dblGATA and control mice received an i.n. instillation of a low dose $(5 \mu \mathrm{g})$ of a clinically relevant mixture of HDM. Five days after HDM injection, 
A Living singlet CD $45.2^{+}$lung cells from naive mice

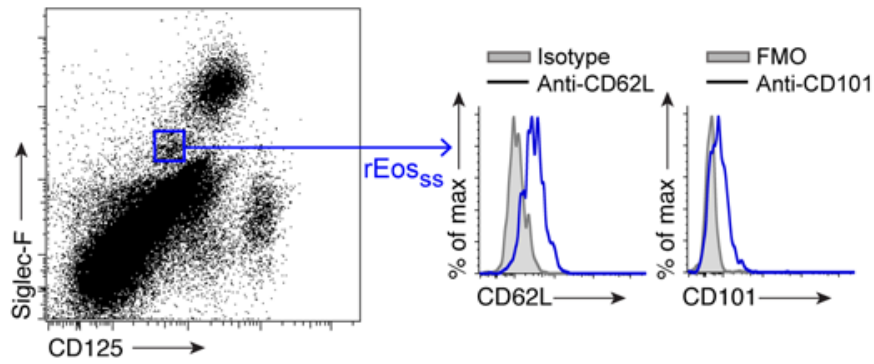

B Living singlet CD $45.2^{+}$lung cells from HDM-treated mice

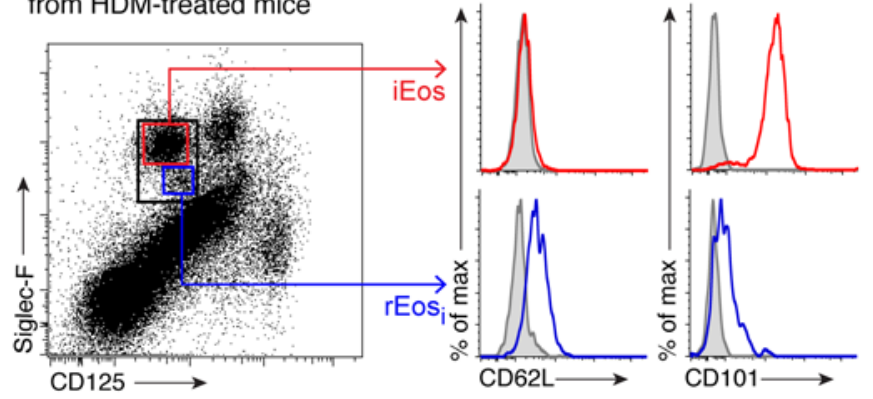

C
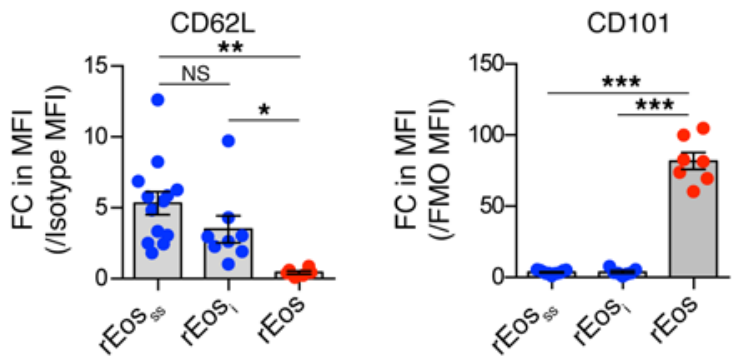

D
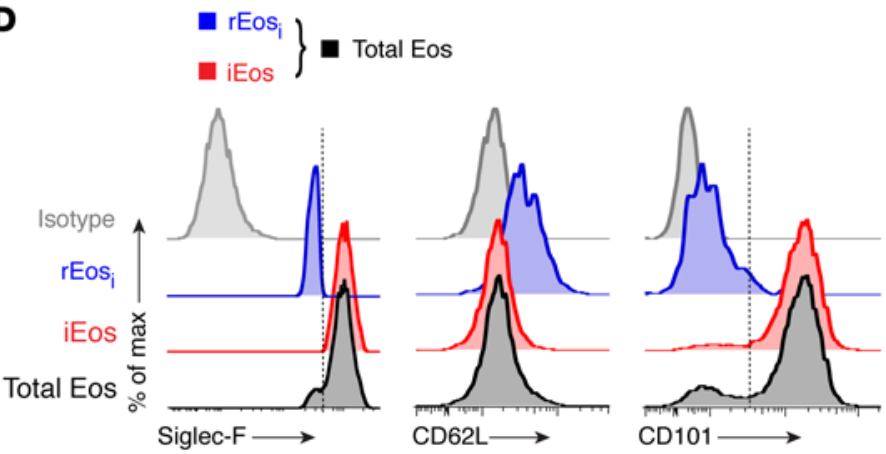

mediastinal lymph node (MLN) cell proliferation and cytokine production following in vitro restimulation with HDM were assessed (Figure 7A). Interestingly, MLN cells from HDM-treated $\triangle$ dblGATA mice proliferated significantly more and produced more of the Th2-associated cytokines IL-4, IL-5, and IL-13 as compared with MLN cells from HDM-treated control mice (Figure 7, B and C). Since $5 \mu$ i.n. HDM exposure also promoted a weak innate recruitment of iEos (i.e., Siglec-F $\mathrm{F}^{\mathrm{hi}} \mathrm{CD} 101^{\mathrm{hi}}$ cells) in the lung (albeit 300 times less significant than the recruitment observed in HDM-allergic mice, Supplemental Figure 11), we assessed the potential contribution of such iEos in Th2 sensitization by treating WT mice i.p. with $\alpha-\mathrm{IL}-5$
Figure 4. Identification of discriminating surface markers for mouse lung rEos and iEos. (A and B) Representative flow cytometric histo-

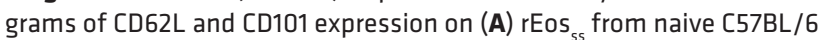
mice and (B) rEos and iEos from HDM-treated allergic C57BL/6 mice. (C) Quantification of CD62L and CD101 expression levels on the surface of $\mathrm{rEos}_{\mathrm{ss}}, \mathrm{rEos}$, and iEos, expressed as the fold change (FC) increase in mean fluorescence intensity (MFI) as compared with the control $\mathrm{MFI}$. Data represent the mean \pm SEM as well as individual values and are pooled from 3 to 5 independent experiments ( $n=7-13 /$ group). (D) Histograms of CD62L and CD101 expression on rEos, (blue) and

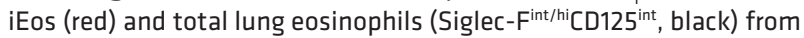
HDM-treated allergic [57BL/6 mice. (C) ${ }^{*} P<0.05,{ }^{* *} P<0.01$, and ${ }^{* *} P<0.001$, by 1 -way ANOVA, followed by Tukey's test for multiple comparisons. FMO, fluorescence minus 1.

Abs (i.e., a regimen that has been shown to inhibit the recruitment of iEos into the lungs without affecting rEos numbers [see Figure 6B and Supplemental Figure 11]) 1 hour before sensitization. As shown in Figure 7, B and C, $\alpha$-IL-5-treated iEos-depleted WT mice were not more susceptible to HDM-induced Th2 responses than were isotype-treated control mice. These results demonstrated that rEos, but not iEos, mediate the increased susceptibility to HDM observed in $\triangle$ dblGATA mice. Intranasal sensitization with a high dose of HDM, which likely bypass innate immune regulatory mechanisms in the lung, induced similar MLN cell responses between $\triangle$ dblGATA and control mice (Supplemental Figure 12), in agreement with previous findings $(59,60)$. These results show that a lack of rEos is associated with increased Th2 sensitization to low doses of allergens and support the idea that rEos can exert homeostatic functions, either by inhibiting $\mathrm{T}$ cells directly or by impairing the ability of antigen-presenting cells to induce Th2 responses. We assessed the effects of $\mathrm{rEos}$ on $\mathrm{T}$ cells and showed that, when cocultured with activated $\mathrm{T}$ cells in vitro, rEos did not impact the ability of $\mathrm{T}$ cells to secrete Th2 cytokines (Supplemental Figure 13A), suggesting that they do not act on $\mathrm{T}$ cells directly. We also found that, unlike conventional DCs, rEos were not able to migrate to the MLNs following treatment with $5 \mu \mathrm{g}$ HDM (Supplemental Figure 13, B and C). Since rEos are located in the lung parenchyma (Figure 1, F and G), i.e., in the vicinity of DCs (55), we postulated that they may contribute to lung homeostasis by inhibiting DC function locally, as reported previously by our group for lung interstitial macrophages (IMs) (55). rEos, unlike IMs, were not able to secrete IL-10 upon LPS stimulation (Supplemental Figure 13D), but were able to inhibit the maturation of antigen-pulsed in vitro-cultured BM-derived DCs (BMDCs). Indeed, when BMDCs were cocultured with FACS-sorted rEos and pulsed with OVA (i.e., grade V OVA contaminated with a low dose of LPS [OVA $\left.{ }_{\mathrm{LPS}}\right]$ ), OVA $\mathrm{OPS}_{\mathrm{LP}}$-BMDS cocultured with $\mathrm{rEos}$ exhibited significantly decreased surface expression of the costimulatory molecules CD80 and CD86 as compared with $\mathrm{OVA}_{\mathrm{LPS}}$-BMDCs alone (Figure 7D), confirming that rEos can inhibit DC maturation. Conversely, neither FACS-sorted lung iEos nor Int Eos were able to inhibit the maturation of cocultured DCs (Figure 7D and Supplemental Figure 14, respectively). To test whether rEos can also affect DC proallergic functions, $\mathrm{OVA}_{\mathrm{LPS}}$-pulsed BMDCs cocultured with or without rEos were then injected i.t. into naive recipient mice. $\mathrm{OVA}_{\mathrm{LPS}}$-BMDCs cocultured with AMs (i.e., a negative control) (55), IMs (i.e., a positive control) (55), or iEos were also ana- 
A Living singlet CD $45.2^{+}$blood cells from naive mice

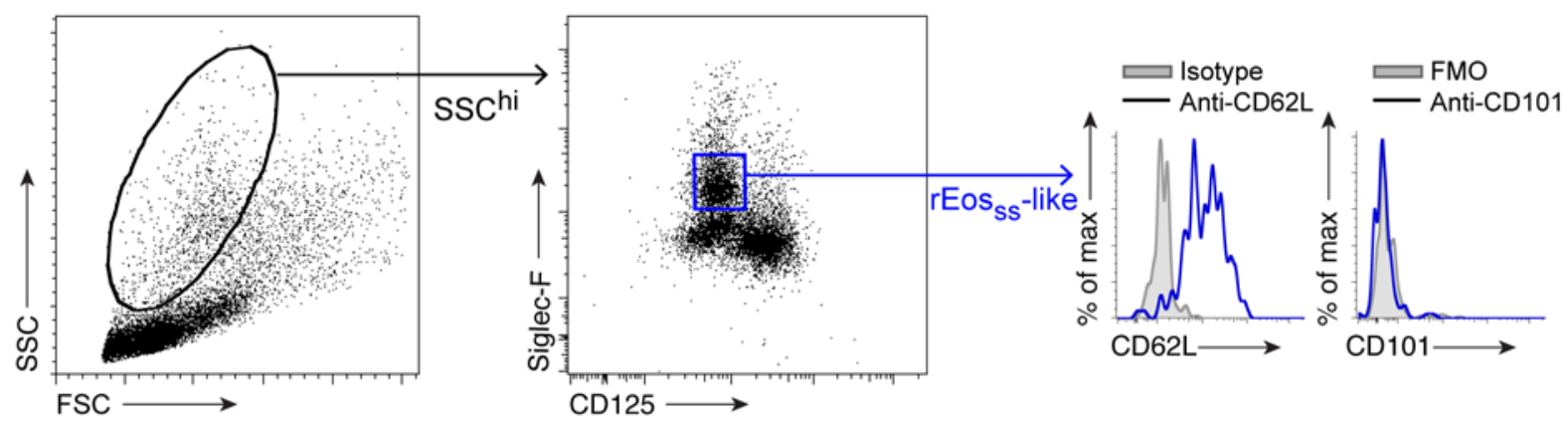

B Living singlet CD $45.2^{+}$blood cells from HDM-treated mice
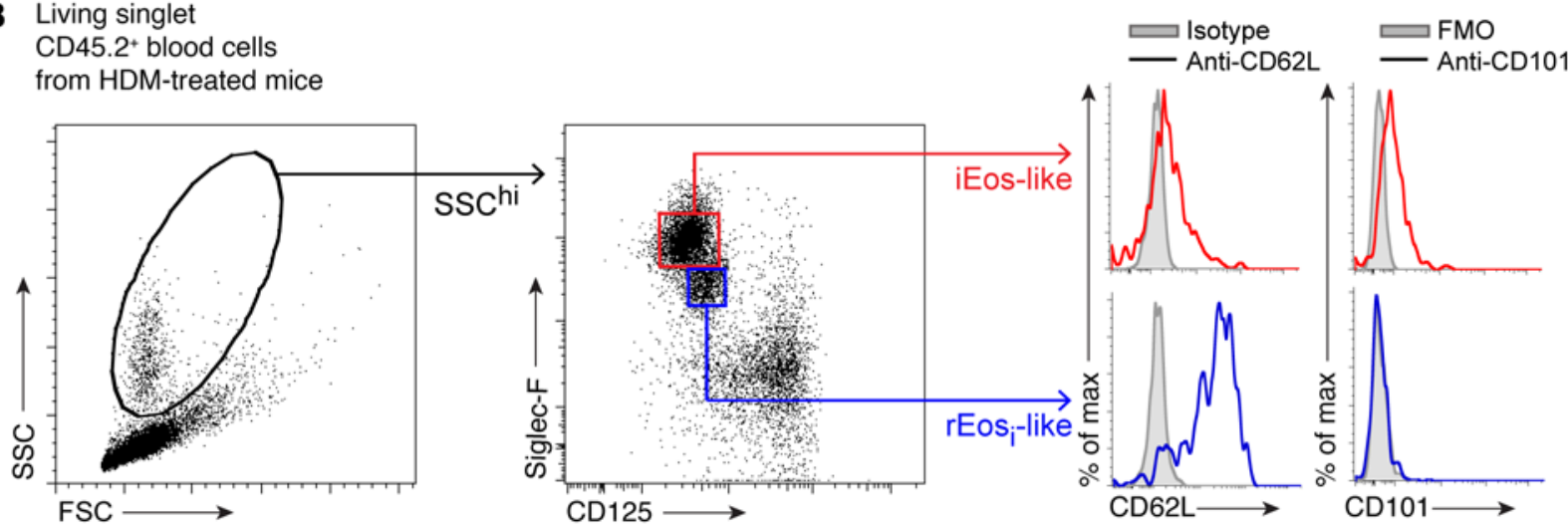

Figure 5. Identification of rEos-like and iEos-like eosinophils in the blood of naive and HDM-treated C57BL/6 mice. White blood cells from (A) naive and (B) HDM-treated allergic C57BL/6 mice were analyzed by flow cytometry (see Figure 2A for experimental outline). Living singlet CD45.2+5SChi cells were analyzed according to Siglec-F and CD125 expression, and representative flow cytometric histograms of CD62L and CD101 expression in the gated populations are shown. Data shown are representative of 1 of more than 8 mice analyzed from 3 independent experiments, each of them giving similar results. FMO, fluorescence minus 1; FSC, forward scatter; SSC, side scatter.

lyzed. Ten days after adoptive transfer, mice were challenged with OVA aerosols to induce airway allergic inflammation (Figure 7E). As expected, mice that received $\mathrm{OVA}_{\mathrm{LPS}}-\mathrm{BMDCs}$ or $\mathrm{OVA}_{\mathrm{LPS}}-\mathrm{BMDCs} /$ AMs, but not those injected with PBS-BMDCs, developed eosinophilia in the BALF, but such eosinophilia was markedly attenuated in mice that received $\mathrm{OVA}_{\mathrm{LPS}}$-BMDCs cocultured with $\mathrm{rEos}$, but not those cocultured with iEos (Figure 7F). In addition, $\mathrm{T}$ cell proliferation and Th2 cytokine production of bronchial lymph node (LN) cells restimulated in vitro with OVA were substantially decreased in mice sensitized with OVA $_{\mathrm{LPS}}$-BMDCs cocultured with $\mathrm{rEos}$ compared with those sensitized with OVA $_{\text {LPS }}$-BMDCs alone or cocultured with AMs or iEos and reached levels similar to those measured in mice sensitized with unpulsed BMDCs (Figure 7, G and H). Similar immunosuppressive effects on DC maturation and function were observed with blood rEos-like cells cocultured with OVA LPS $_{-B M D C s}$ (Supplemental Figure 15).

Together, these results show that a) at the time of exposure to a low dose of HDM, the absence of rEos, but not the absence of iEos, results in increased Th2 sensitization to HDM, and that 2) rEos, as opposed to iEos, are able to inhibit the proallergic functions of DCs in vitro, thereby preventing the development of DC-induced airway allergy.
Lung parenchymal eosinophils exist in humans and are phenotypically distinct from iEos found in asthmatic airways. To assess the human relevance of our findings, we examined Congo redand MBP-stained lung sections from 5 healthy human donors and 5 asthmatic patients (Figure 8A and Supplemental Table 1) and quantified the numbers of eosinophils found in the lung parenchyma and peribronchial area (Figure 8B). As in mice, we detected eosinophils in similar numbers in the lung parenchyma of healthy donors and asthmatic subjects (Figure 8, A and B and Supplemental Figure 16A), supporting the existence in humans of lung parenchymal rEos whose numbers seemed unaffected by lung inflammation. Additional eosinophils were also found in the peribronchial area of asthmatic lungs, probably representing classical iEos infiltrating the bronchi (Figure 8, A and B). Of note, by assessing the average numbers of rEos per microscopic field in mouse and human lung sections (see Figure 2I [mouse] vs. Figure $8 \mathrm{~B}$ [human]), our data support the idea that the density of the rEos population is comparable in mouse and human lungs.

To further investigate the potential existence of distinct eosinophil subsets in humans, we assessed the surface expression of Siglec-8, CD62L, and CD101 (i.e., the human orthologs of the discriminating surface markers found in the mouse) on 2 populations 
A

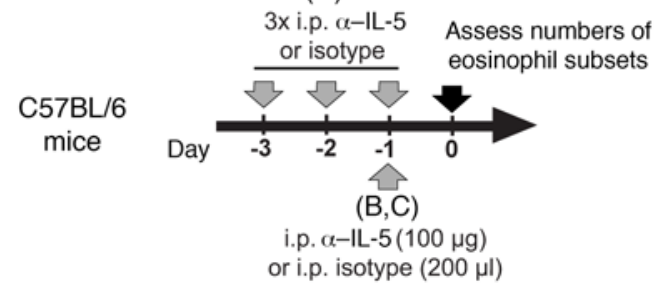

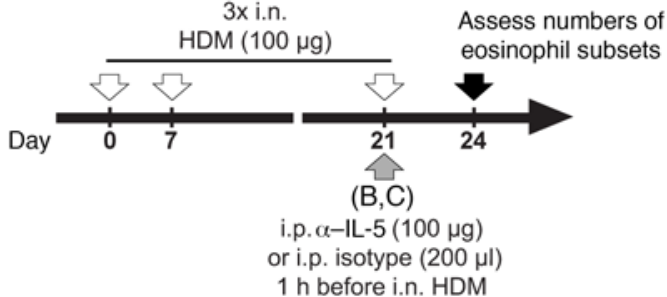

B Living singlet CD45.2+ lung cells

Lung
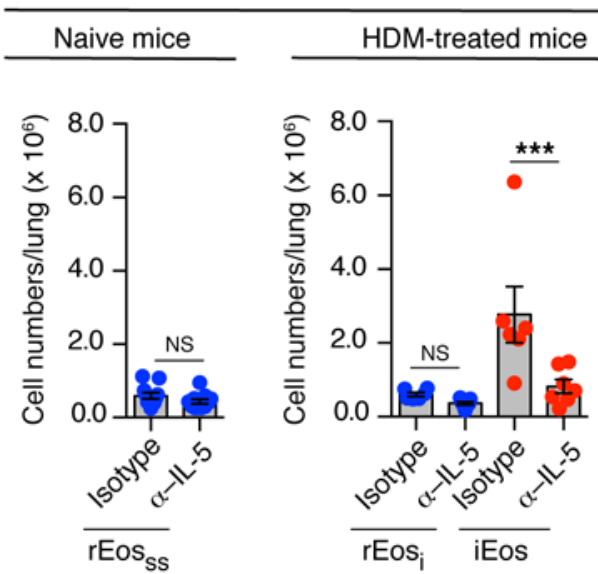

- rEos (Siglec-Fint CD125 $\left.{ }^{\text {int }} \mathrm{CD} 101^{10}\right)$

- iEos (Siglec-Fi ${ }^{\mathrm{n}} \mathrm{CD} 125^{\mathrm{in}} \mathrm{CD} 101^{\mathrm{in}}$ )
Living singlet SSChi CD45.2+ blood cells

Blood

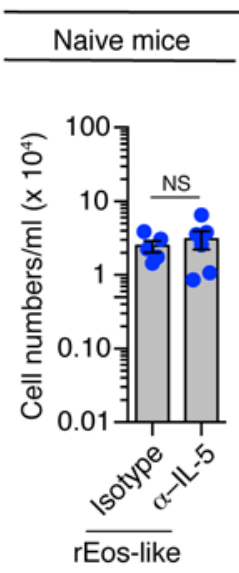

D Living singlet CD45.2+ lung cells

$\frac{\text { Lung }}{\text { Naive mice }}$

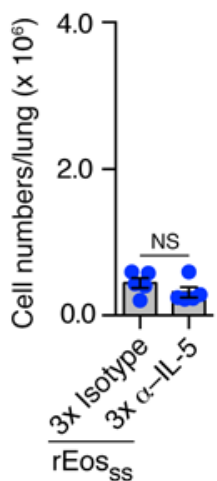

- rEos-like (Siglec-Fint CD125 ${ }^{\text {int }} C D 101^{-}$)

- iEos-like (Siglec-Fin CD125 $5^{\text {int } C D 101+)}$

$\mathbf{E}$

Phosphorylation of ERK1/2 (p-ERK) on lung primary rEos and iEos $5 \mathrm{~min}$ after vehicle or rlL-5 treatment in vitro

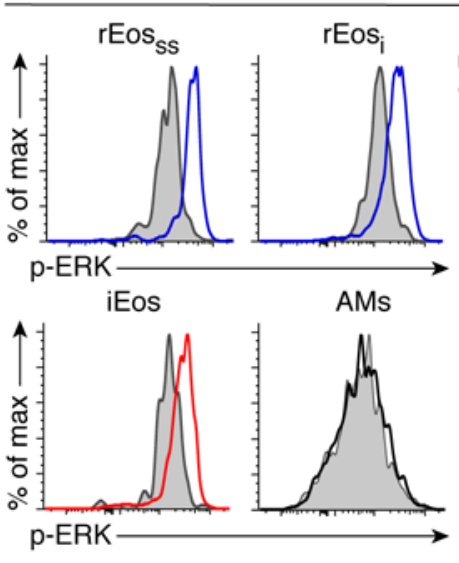

Vehicle

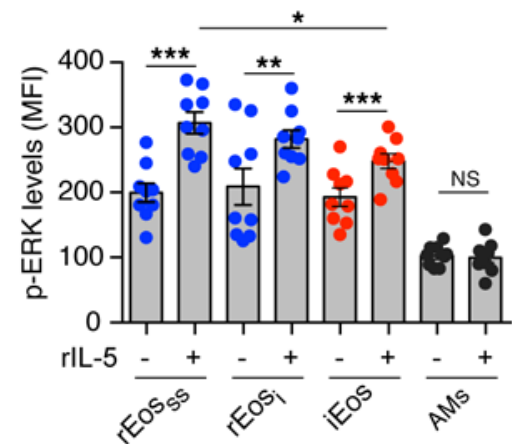

- rlL-5 $(50 \mathrm{ng} / \mathrm{mL})$
F Survival of lung primary rEos and iEos after vehicle or rlL-5 treatment in vitro

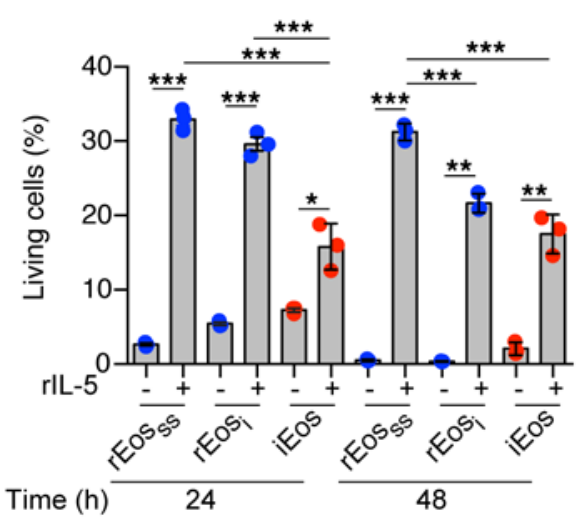

Figure 6. Sensitivity and responsiveness of eosinophil subsets to in vivo $\alpha-I L-5$ and in vitro rlL-5 treatments. (A) Experimental outline for data shown in B-D. (B-D) Effects of in vivo $\alpha-$ IL-5 (TRFK5) treatment on the numbers of (B and $\mathbf{D}$ ) lung and (C) blood eosinophil subsets in naive and HDM-treated allergic C57BL/6 mice. (E) Phosphorylation of ERK1/2 was assessed by phospho-flow cytometry on freshly isolated eosinophils and AMs stimulated in vitro with rIL-5 or vehicle. Representative flow cytometric histograms and quantification of p-ERK levels. (F) Survival (as assessed by incorporation of 7-AAD) of FACS-sorted lung eosinophil subsets stimulated ex vivo, with or without rlL-5, for the indicated times. More than $90 \%$ of the cells were negative for 7-AAD before stimulation. (B-F) Data represent the mean \pm SEM as well as individual values and are pooled from 2 to 3 independent experiments ( $n=5-11$ group). ${ }^{*} P<0.05$, ${ }^{*} P<0.01$, and ${ }^{* * *} P<0.001$, by 2 -tailed Student's $t$ test (B and $\mathbf{C}$, left panels, and $\mathbf{D}$ ) or a mixed-effects model, followed by Tukey's test for multiple comparisons (B and C, right panels) on log-transformed values and (E and F) by Welch's $t$ test (for comparison of vehicle vs. IL-5-treated groups) or 1-way ANOVA, followed by Tukey's test for multiple comparisons (for comparison of IL-5-treated subsets). cpm, counts per minute. 
A

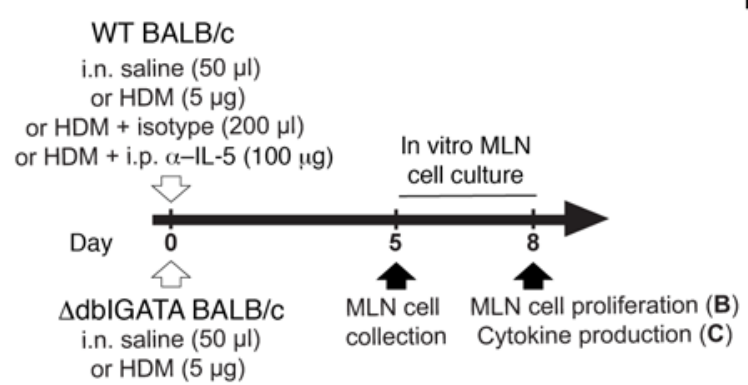

C
WT- Sal
WT - HDM + Iso
$\triangle d$ dbIGATA - Sal
WT - HDM
WT - HDM $+\alpha-$ IL-5

B
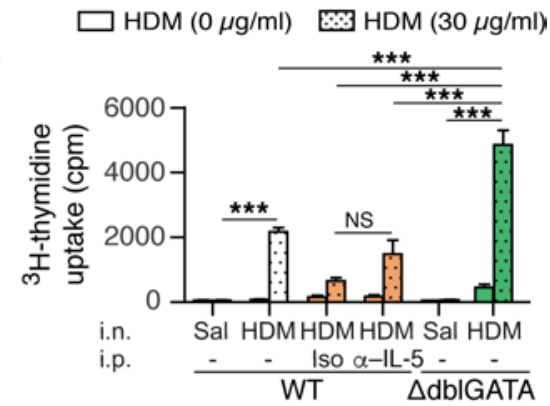

D Maturation of OVA-pulsed

BMDCs cocultured with rEos or iEos
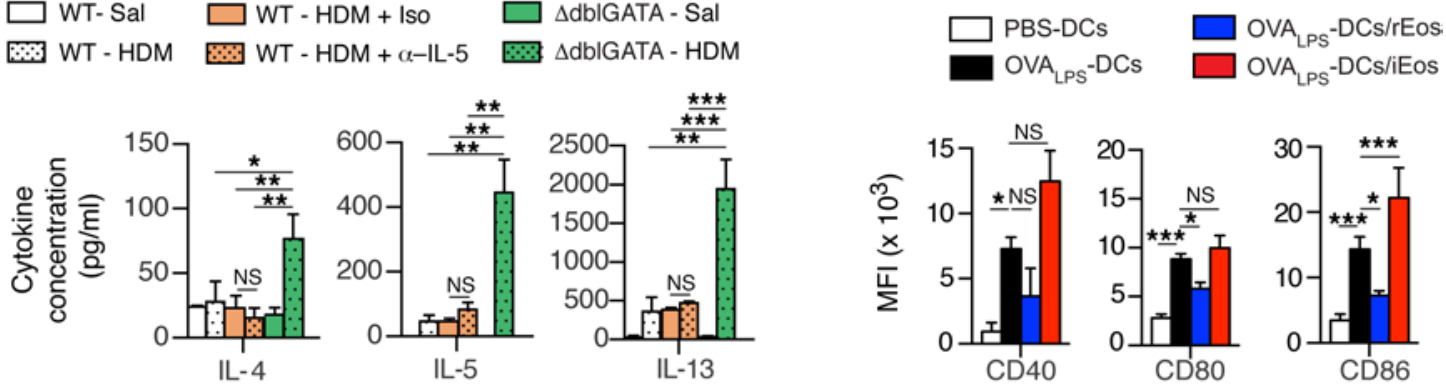

$\mathbf{F}$

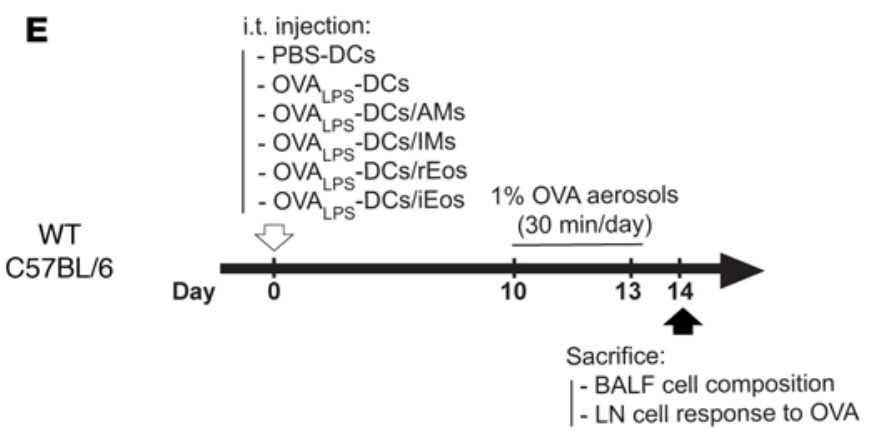

G

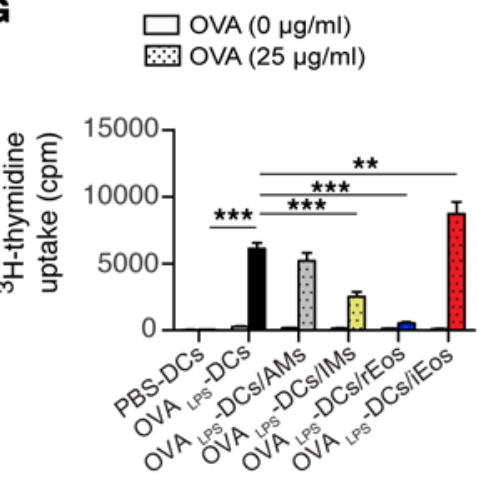

H $\square$ PBS-DCs $\square$ OVA $_{L P S}$-DCs/IMs

$\square$ OVA $_{\text {LPS }}$-DCs $\square$ OVA $_{\text {LPS }}$-DCs/rEos

$\mathrm{OVA}_{\mathrm{LPS}}$-DCs/AMs $\square$ OVA $_{\text {LPS }}$-DCs/iEos
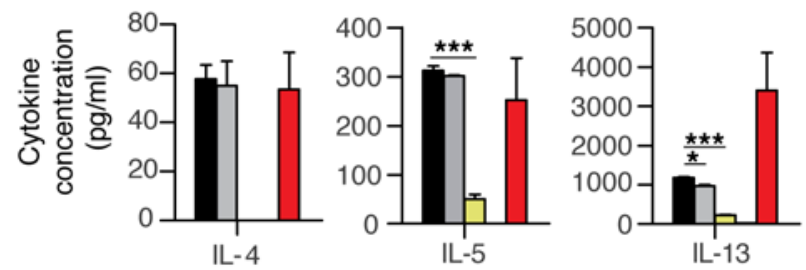

Figure 7. Assessment of rEos immunosuppressive functions using eosinophil-deficient $\triangle$ dbIGATA mice and DC coculture experiments. (A) Experimental outline for $\mathbf{B}$ and $\mathbf{C}$. Briefly, the indicated groups of mice were sensitized i.n. with $5 \mu$ g HDM or injected with saline, and the MLN cell response to HDM was assessed 5 day later. Some WT mice also received an i.p. injection of $\alpha-$ IL- 5 or isotype Abs 1 hour before HDM instillation. (B) Proliferation of MLN cells restimulated for 3 days with or without HDM. (C) Cytokine concentrations in culture supernatants of MLN cell cultures restimulated with

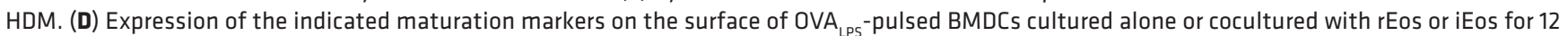
hours. (E) Experimental outline for F-H. (F) Total and eosinophil cell counts in the BALF. (G) Proliferation of LN cells restimulated for 3 days with or without OVA. (H) Cytokine concentrations in culture supernatants of LN cell cultures restimulated with OVA. Data shown represent the mean \pm SEM and were (B and $\mathbf{C}$ ) pooled from 3 independent experiments ( $n=6$ biological replicates/group) and ( $\mathbf{D}-\mathbf{H})$ are representative of 1 of 4 different batches of BMDCs cocultured with primary rEos, AMs, or IMs and 1 of 3 different batches of BMDCs cocultured with primary rEos or iEos isolated from independent cohorts of mice. ${ }^{*} P<0.05,{ }^{*} P<0.01$, and ${ }^{* *} P<0.001$, by 2 -way ANOVA (B, F, and $\mathbf{C}$ ) or 1-way ANOVA (C, $\mathbf{D}$, and $\left.\mathbf{H}\right)$, followed by Tukey's test for multiple comparisons. Iso, isotype Abs; Sal, saline. 
of human primary eosinophils (Figure 8C and Supplemental Figure 16B): a) eosinophils freshly isolated from normal lung tissue (rEos) (Supplemental Table 2), with more than $90 \%$ of eosinophils residing in the parenchyma (see also Supplemental Methods) and b) iEos found in the sputa of asthmatic subjects (Supplemental Table 3) (Figure 8C). rEos and iEos could not be distinguished from each other on the basis of morphology (Figure 8C) or on the basis of surface levels of Siglec-8 and CD101 (Figure 8, D and E). However, interestingly, iEos were consistently found to be negative for CD62L expression, whereas rEos were expressing variable levels of CD62L, reminiscent of what we observed in mice (Figure 8, D and E). In search for a marker that, like CD101 in mice, was upregulated in iEos, but not rEos, we assessed the surface expression of several markers whose mRNA levels have been shown to be upregulated in blood eosinophils from asthmatic subjects as compared with those from healthy controls (61). Among the candidates tested, we found that expression of IL-3R was significantly higher in iEos compared with that detected in rEos (Figure 8, D and E). While based on a limited number of samples analyzed, our data support the existence of positionally and phenotypically distinct subsets of lung eosinophils in humans and identify IL-3R and, to a lesser extent, CD62L as 2 promising markers allowing discrimination between such lung rEos and iEos.

\section{Discussion}

Lung rEos constitute one abundant but little-characterized population of tissue eosinophils (26). Here, we showed that pulmonary rEos express intermediate levels of Siglec-F and CD125, two nonspecific markers of eosinophils $(30,31)$. Siglec-F was also found to be expressed by AMs, a finding consistent with that of previous studies $(28,29)$, and by lung neutrophils, which has not been reported before. Although CD125 expression is thought to be restricted to eosinophils and B-1 cells in mice $(28,29,62-64)$, we unexpectedly found that lung neutrophils expressed high levels of CD125, suggesting that these cells may also be influenced by IL-5. Supporting this finding, mouse neutrophils have been shown to migrate in response to rIL-5 treatment (65). These findings are particularly important in light of the numerous mouse studies, in which eosinophils were identified on the basis of their high granularity and expression of Siglec-F and/or CD125 and in which a possible contamination with neutrophils may have led to misinterpretations.

Microscopy revealed that lung rEos have a ring-shaped nucleus, which has been reported recently (25). The presence of such a nucleus in mouse eosinophils is considered a sign of cell immaturity $(66,67)$, suggesting that pulmonary rEos retain an immature phenotype when spreading into the lungs. Using histochemistry, we further showed that lung rEos were located in the parenchyma, which contrasts with the localization of eosinophils in asthma, in which eosinophils are classically thought to infiltrate the peribronchial area (68). Using electron microscopy, we next showed that rEos exhibited signs of piecemeal degranulation, suggesting that they are actively secreting their granule contents at steady state. Another interesting feature of lung rEos was their ability to phagocytose fluorescent beads, suggesting a scavenger function for these cells. Given that fluorescent beads were unable to reach the circulation, this experiment also definitively proved that pulmonary rEos were located in the tissue rather than in the blood vessels. The tis- sue localization of lung rEos was also evidenced by the fact that the number of rEos in the lung exceeded $4 \times 10^{5}$, which corresponds to 2 times the number of eosinophils present in the entire blood circulation of C57BL/6 mice $(69,70)$. Finally, time-course studies showed that rEos were not present in the lung at birth but gradually increased in number to reach a maximal density by day 7. This is in agreement with a previous report showing that murine thymic rEos reach a plateau by 2 weeks of age (22). These data tend to link the colonization of the lung by rEos with the development of the microbiota, but this needs further investigation.

Over the past few decades, most of the immune cell populations have been divided into different subpopulations with specific phenotypes and functions. However, it is still believed that inflammatory and homeostatic eosinophils are the same cells in different activation states. Although it cannot be ruled out that rEos may transform into iEos, our work provides evidence that lung $r$ Eos and iEos represent distinct terminally differentiated eosinophil subsets. First, the phenotypic features of rEos remained unchanged and differed from those of iEos during allergic airway inflammation. Siglec- $F^{\text {int }} \mathrm{CD} 125^{\text {int }} \mathrm{CD} 62 \mathrm{~L}^{+} \mathrm{CD} 101^{\text {lo }} \mathrm{rEos}$ cohabited with iEos, which were defined as Siglec- $F^{\text {hi }} \mathrm{CD} 125^{\text {int }} \mathrm{CD} 62 \mathrm{~L}^{-} \mathrm{CD} 101^{\text {hi }}$ cells with a segmented nucleus. Microarray analyses further revealed that rEos and iEos had substantially different gene expression signatures, but that not a single gene was differentially expressed at a significant level $(P<0.01, \mathrm{FC}>2)$ between $\mathrm{rEos}_{\mathrm{ss}}$ and $\mathrm{rEos}_{\mathrm{i}}$. Second, rEos and iEos were confined to different compartments of the lung. iEos were localized in the peribronchial areas, whereas rEos resided in the parenchyma, even during allergic inflammation. As the inflammation is thought to be mainly restricted to the peribronchial connective tissue in asthma (68), this observation could account for the fact that rEos were not at all affected by the inflammatory process. Third, we did not find any continuity between the rEos and iEos subsets. As rEos displayed an immature phenotype, attested by their ring-shaped nucleus, it is tempting to speculate that these cells are precursors of iEos. However, if this were true, cells having an intermediate state between $\mathrm{rEos}_{\mathrm{ss}}$ and iEos should have been detected in HDM-challenged asthmatic mice. For example, $\mathrm{rEos}_{\mathrm{i}}$ should have displayed an intermediate gene expression profile or an intermediate morphology compared with $\mathrm{rEos}_{\mathrm{ss}}$ and iEos, which was not the case. The only apparent continuity between rEos and iEos was that Siglec-F expression was only slightly increased on iEos compared with that observed on rEos, supporting the idea put forth by Abdala Valencia and colleagues (25), in which rEos can increase their expression of Siglec-F to become iEos during the inflammatory process. However, when analyzed for their expression of CD101, one of the most discriminating markers identified by microarrays, the $\mathrm{rEos}_{\mathrm{i}}$ and iEos subpopulations did not overlap at all. Finally, rEos- and iEos-like eosinophils were found in the blood, suggesting that the differentiation of both cell subsets mainly occurs before their recruitment to the lungs.

Mice lacking functional GATA1 were devoid of lung rEos, demonstrating that these cells develop in the BM from eosinophil progenitors. Here, we provided evidence that rEos and rEos-like cells are not dependent on IL-5 for their presence in the lungs and the blood, respectively. Nevertheless, we also showed that $\mathrm{rEos}$ express the receptor for IL- 5 and can respond to IL-5 in 
A
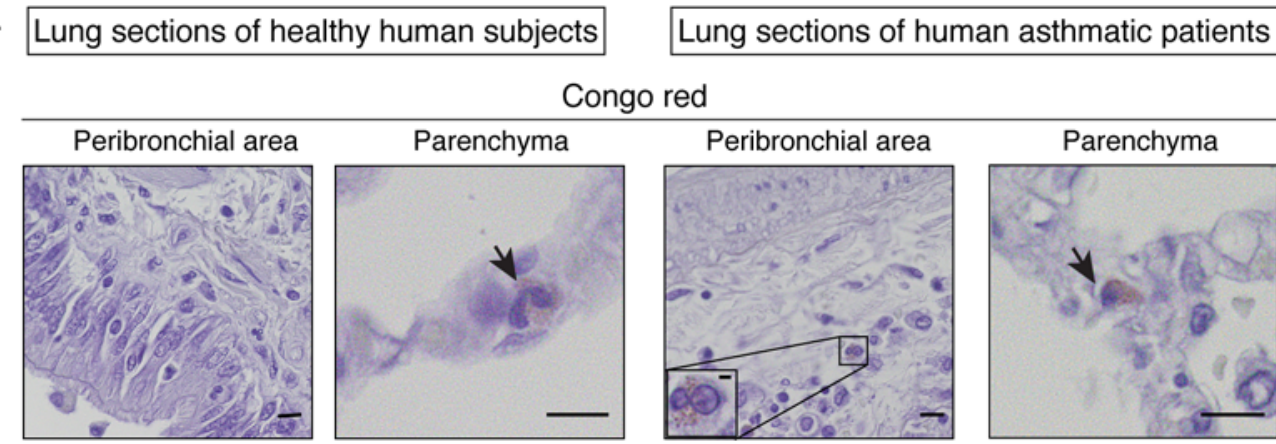

MBP
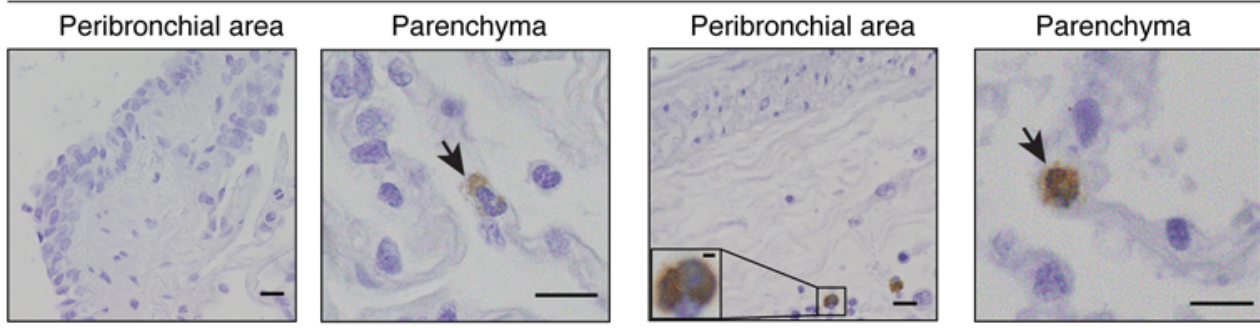

B

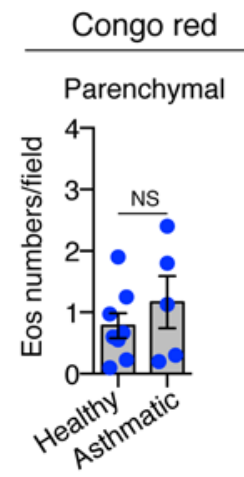

Peribronchial

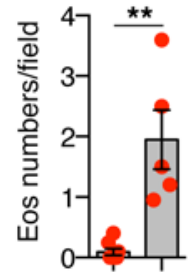

Heging

C

Living singlet CD45+SSChi $\mathrm{CD} 11 \mathrm{~b}$-enriched cells isolated from:

Normal lung tissue

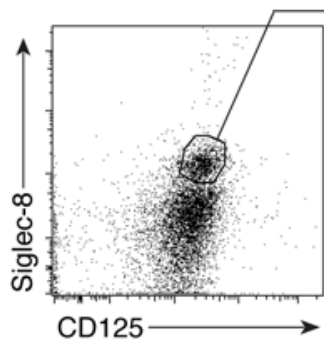

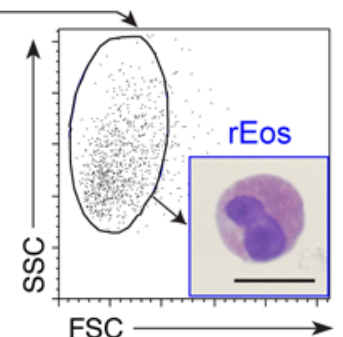

FSC
Sputum of eosinophilic asthmatic patients
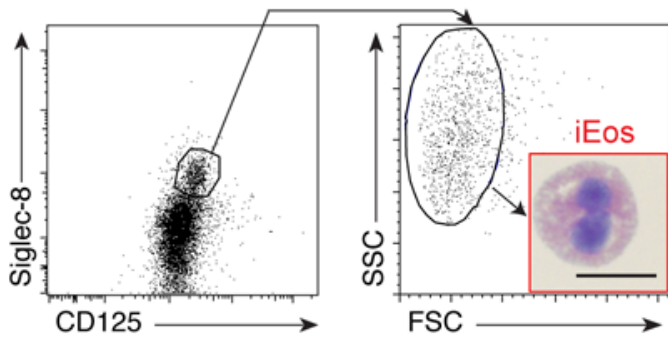
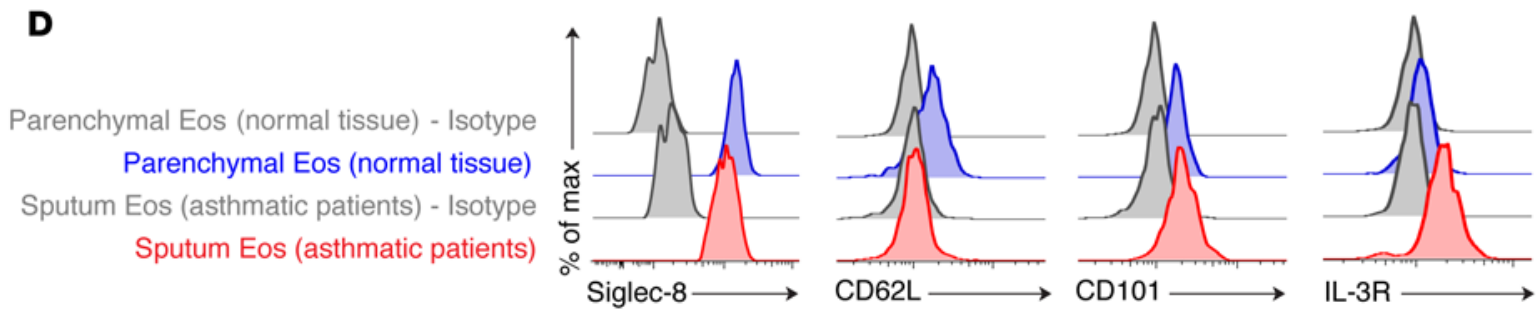

E rEos : Parenchymal eosinophils from normal lung tissue

- iEos : Sputum eosinophils from asthmatic patients
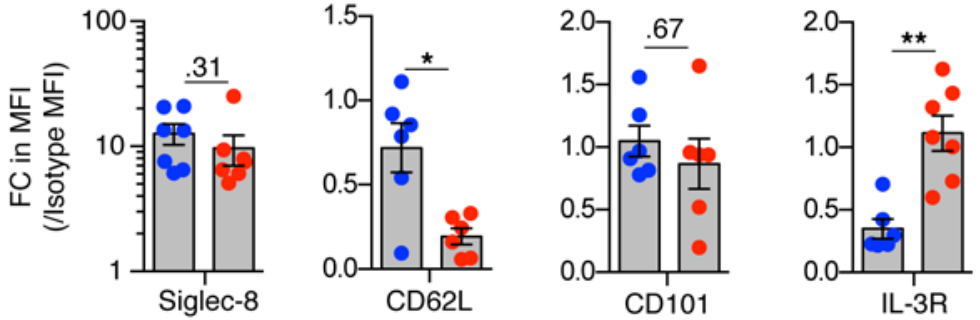
Figure 8. Localization, morphology, and phenotype of lung rEos and iEos in humans. (A) Representative Congo red- and MBP-stained lung sections of healthy human donors and asthmatic patients. Arrows indicate Congo red- and MBP-positive eosinophils, respectively. (B) Quantification of parenchymal and peribronchial eosinophils in Congo red-stained lung sections. Eos, eosinophils. (C-E) Parenchymal eosinophils from normal lung tissue (rEos) and eosinophils from the sputa of eosinophilic asthmatic patients (iEos) were analyzed morphologically and phenotypically. (C) Dot plots of living singlet CD45 ${ }^{+}$SCC $^{\text {hi }}$ cells according to surface Siglec- 8 and CD125 expression. Insets depict a representative photograph of FACS-sorted rEos and iEos. (D) Representative flow cytometric histograms of Siglec-8, CD62L, CD101, and IL-3R expression on rEos and iEos. (E) Quantification of Siglec-8, CD62L, CD101, and IL-3R expression levels on the surface of rEos and iEos, expressed as the FC increase in MFI as compared with the control MFI. Number are the $P$ values for comparisons that were not significant $(P>0.05$ ). (B and $\mathbf{E})$ Data shown represent the mean \pm SEM as well as individual values. Each dot represents a single individual analyzed ( $n=5$-8/group) (see also Supplemental Methods and Supplemental Tables $1-3)$. ${ }^{*} P<0.05$ and ${ }^{* *} P<0.01$, by nonparametric Mann-Whitney $U$ test. Scale bars: $10 \mu \mathrm{m}$ and $2 \mu \mathrm{m}$ (insets in $\mathbf{A}$ ).

vitro. The biological effects of IL-5 on rEos, however, remain to be determined. One likely scenario is that lung rEos, as opposed to iEos, are not affected by IL-5 during allergen-induced inflammation, because they are not reached by IL-5 as a result of their specific parenchymal localization. It is indeed thought that IL-5secreting cells such as effector Th2 cells are mainly infiltrating the peribronchial area $(71,72)$, where rEos are absent. Supporting this notion, transcriptomic analyses of rEos showed no differentially expressed genes between steady state and the effector phase of allergic inflammation, at a time when IL-5 is increased in the lung. In this context, if rEos were under the influence of IL-5, we would have expected to find differentially expressed genes between $\mathrm{rEos}_{\mathrm{ss}}$ and $\mathrm{rEos}_{\mathrm{i}}$ as a result of IL-5 exposure. Of note, such an IL-5 signature was present in iEos, which upregulated several IL-5-dependent genes such as Bcl6, Ccnd2, and Sema $4 a$, as com-

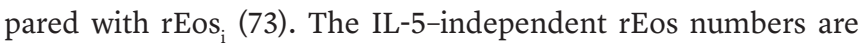
also reminiscent of the results from Kopf et al. (52), who reported that the basal numbers of blood eosinophils are only moderately reduced in IL-5-deficient mice, which are incapable of developing eosinophilia when infected with helminths or challenged with allergens $(52,74)$. Also in accordance with our results, a population of subepithelial $\mathrm{rEos}$ has been shown to still be present in the uterus of IL-5-deficient mice. All these observations are consistent with a model in which blood rEos-like eosinophils are produced by default in the absence of IL-5. These IL-5-independent eosinophils, although terminally differentiated, display an immature phenotype and rapidly home to the tissues where they exert homeostatic functions. In this model, IL-5 acts as an emergency cytokine that amplifies the production of eosinophils and skews most of the newly generated eosinophils toward the inflammatory phenotype. These iEos, which have a more mature phenotype, are then recruited to the sites of $\mathrm{Th} 2$ cell responses $(71,72)$, where they participate in the elimination of extracellular parasites and the exacerbation of inflammation. The eosinophils that are not directed to the inflammatory phenotype upon IL-5 stimulation contribute to the maintenance of the pool of homeostatic eosinophils. Such a model, if applicable to humans, could further explain why residual eosinophils are found in the lung (75), duodenal mucosa $(76)$, or blood $(77,78)$ of eosinophilic patients treated with humanized $\alpha-I L-5$ Abs (75-78).

Under basal conditions, most eosinophils reside in the tissue where they exert key homeostatic functions $(1,2,8,16)$. Microarray analyses revealed that lung rEos specifically expressed several genes, such as Anxa1 (46), Nedd4 (47), Runx3 (48), Serpinb1a (49), and $L d l r$ (50), that are involved in the maintenance of lung immune homeostasis. Some of these genes are implicated in the negative regulation of $\mathrm{Th} 2$ cell responses in the lung. Indeed, mice deficient for annexin A1, a phospholipase A2 inhibitor encoded by the Anxal gene, or for RUNX3, the transcription factor encoded by the Runx 3 gene, spontaneously develop asthma-like diseases (46, 48). Moreover, mice lacking the low-density lipoprotein receptor, the product of $L d l r$, are more prone than are WT controls to developing airway hyperreactivity when sensitized to allergens (50). All these observations prompted us to investigate whether lung rEos, which overexpressed those genes, were endowed with the capacity to prevent airway allergy. Using a model of sensitization to a low dose of HDM, we showed that rEos-deficient $\triangle$ dblGATA mice, as opposed to $\alpha$-IL-5-treated iEos-depleted WT mice, exhibited increased sensitivity to HDM compared with control mice, thereby confirming our hypothesis. This unexpected immunosuppressive function of lung rEos strikingly contrasts with the known proinflammatory and pro-Th2 roles of pulmonary iEos $(1,8,12,13$, $15,36,59,79)$ and further reinforces the idea that these eosinophil subsets are functionally different.

Our results are in apparent contradiction with those of previous studies that also used eosinophil-deficient mice, suggesting that eosinophils are not influencing the sensitization phase of OVA- or HDM-induced airway allergy $(59,60)$. However, in these studies, the authors used either a model of systemic sensitization with OVA and the pro-Th2 adjuvant alum or models of i.n. sensitization with high doses of HDM (i.e., $2 \times 50 \mu \mathrm{g}$ or $5 \times 25 \mu \mathrm{g}$ ). At these doses, which are far above the common environmental HDM concentrations, sensitization occurred in $100 \%$ of the mice. Our hypothesis is that supraenvironmental doses of HDM trigger potent pro-Th 2 mechanisms that bypass the innate immune regulatory mechanisms of the lung, as it is also the case with alumbased sensitization. This notion is also supported by our observation that a single i.n. exposure of mice to $100 \mu \mathrm{g}$ HDM induced similar Th2 cell responses in both $\triangle \mathrm{dblGATA}$ and control mice.

No eosinophils were found in the MLNs following stimulation with low doses of HDM, demonstrating that, under these conditions, rEos are unable to migrate to the draining LNs and therefore must act locally to weaken allergic sensitization. This is not surprising given that the other innate immune cells known to control lung Th2 responses are also located in the parenchyma. Indeed, plasmacytoid DCs, which play a key role in limiting allergic sensitization in the airways, are parenchymal cells (80). Moreover, lung IMs, which are in close contact with pro-Th2 DCs in the parenchyma, have the ability to prevent DC-mediated Th2 sensitization in an IL-10-dependent manner (55). We showed here that lung rEos were not able to produce IL-10 but, like IMs, could impact DC maturation in vitro and the ability of 
DCs to induce a Th2 response. Importantly, this effect was specific for rEos, as iEos were not able to inhibit the maturation and pro-Th2 function of DCs. Although microarray analyses provided some clues as to how rEos may regulate DC function at the molecular level, further investigations will be needed to elucidate these mechanisms.

Our analyses of small Int Eos, the largest population of steady-state eosinophils (26), supported the idea that these cells are phenotypically and functionally different from lung rEos. First, Int Eos, unlike rEos, did not express CD62L. Second, they were not able to inhibit DC maturation in vitro and even tended to promote such maturation, concordant with the observations from a previous report (14). Third, while Int Eos have been shown to regulate $\operatorname{IgA}^{+}$plasma cells in the gut (17), our data showed that rEos did not influence the numbers of plasma cells in the lung. These results are consistent with the idea that the tissue microenvironment can shape the phenotype and function of rEos according to the specific needs of each organ.

In humans, lung parenchymal eosinophils have rarely been evaluated, although some studies indicate the presence of eosinophils in distal and parenchymal lung sites (81-83) in asthmatic patients $(81,82)$, but also in healthy subjects $(81,83)$. As superficial airways are more easily accessible than are distal lung sites for diagnostic purposes, it is not surprising that there has been a bias in focusing mainly on inflammatory airway eosinophils from endobronchial biopsies and BALF, rather than on parenchymal eosinophils. The present study confirmed previous studies showing that eosinophils were present in the lung parenchyma of healthy humans. Though based on a limited number of subjects analyzed, our data also showed that such parenchymal eosinophils were not more numerous in the lungs of asthmatic patients, in which peribronchial eosinophils were also found. We thus speculate that lung parenchymal eosinophils actually "reside" in the normal lung and represent the human counterpart of mouse rEos. Further supporting this, we have shown that human rEos expressed variable levels of CD62L, as opposed to sputum eosinophils from asthmatic patients, which were found to be negative for CD62L. Last, but not least, we identified IL-3R as a surface marker highly expressed on such iEos. One limitation of the present study is that sputum induction and processing may have modified some parameters of iEos. A better comparator of eosinophils from normal lung tissue would have been eosinophils from lung tissue of asthmatic patients, which would have been expected to contain both Siglec- $8^{+} \mathrm{CD} 62 \mathrm{~L}^{+} \mathrm{IL}-3 \mathrm{R}^{\text {lo }} \mathrm{rEos}$ and Siglec- $8^{+}$CD $62 \mathrm{~L}^{\mathrm{lo}} \mathrm{IL}-3 \mathrm{R}^{\mathrm{hi}}$ iEos. Unfortunately, such material was not available. Nevertheless, our data suggest the existence of at least 2 phenotypically distinct eosinophil subsets in the human lung, i.e., a lung-resident parenchymal subset found in the normal lung and an inflammatory subset recruited to the airways during allergic inflammation. Whether rEos exerts immunosuppressive function in humans remains to be determined and represents an exciting question for future research.

In conclusion, our results indicate that lung rEos are not merely immature cells, but represent a positionally and phenotypically distinct eosinophil subset that participates in the fine tuning of respiratory immune responses by preventing Th2 responses to low doses of allergens, at least in mice.

\section{Methods}

Mice. WT CD45.2 C57BL/6J, WT BALB/c, and $\Delta$ dblGATA (C.CgGata1 $^{\text {tm6Sho }} / \mathrm{J}$ ) mice were purchased from The Jackson Laboratory. All mice were housed and bred in institutional specific pathogen-free facilities. Age-matched females were used at 6 to 10 weeks of age, unless otherwise indicated.

Reagents. Lyophilized HDM (Dermatophagoides farinae) extracts were from Greer Laboratories. Grade III and grade V OVA were from Sigma-Aldrich. $\alpha$-IL-5-neutralizing Abs (clone TRFK5; BD Biosciences) or control isotype Abs (rat IgG $_{1}$ ) were from BD Biosciences. Details regarding additional reagents can be found in the respective sections below and in the Supplemental Methods.

Cell isolation, stainings, and flow cytometry. To obtain single lung cell suspensions, lungs were perfused with $10 \mathrm{ml}$ PBS through the right ventricle, cut into small pieces, and digested for 1 hour at $37^{\circ} \mathrm{C}$ in HBSS containing $1 \mathrm{mg} / \mathrm{ml}$ collagenase A (Roche) and 0.05 $\mathrm{mg} / \mathrm{ml}$ DNase I (Roche).

Staining reactions were performed at $4^{\circ} \mathrm{C}$ after incubation with $2.4 \mathrm{G} 2 \mathrm{Fc}$ receptor Abs to reduce nonspecific binding. Cell phenotyping and cell sorting were performed on a FACSAria III (BD Biosciences). Results were analyzed with FlowJo software (Tree Star). Additional details can be found in the Supplemental Methods.

Cytologic examination. Cytologic examination of FACS-sorted cell populations was performed on cytospin preparations stained with Hemacolor (Merck KGaA). Sections were examined with a BX45 microscope (Olympus), and photographs were taken with a U30 camera (Olympus).

Phagocytic activity. Naive C57BL/6 mice were injected i.t. with 50 $\mu \mathrm{l}$ of $10 \%$ (v/v) $0.5 \mu \mathrm{m}$ fluorescent beads. Six hours later, blood cells, cells from the perfusion liquid of the lung vasculature, and single lung cell suspensions were obtained and analyzed by flow cytometry or confocal microscopy (Leica SP5). Additional details can be found in the Supplemental Methods.

Lung histology and IHC. Methodological details regarding sample processing, histology, and IHC are described in the Supplemental Methods.

HDM-induced airway allergy. Lightly isoflurane-anesthetized WT C57BL/6 mice received an i.n. instillation of vehicle (LPS-free saline) or HDM $(100 \mu \mathrm{g}$ in $50 \mu \mathrm{l})$ on days 0,7 , and 14 . Mice were sacrificed on day 17, and the BALF and lung cells were analyzed.

Microarray data. Microarray data have been deposited in the Array Express public database under accession number E-MTAB-4084 (http://www.ebi.ac.uk/arrayexpress/experiments/E-MTAB-4084).

In vivo $\alpha-I L-5$ treatment. Numbers and phenotype of blood and lung eosinophil subsets from $\alpha$-IL-5-treated (100 $\mu$ g i.p.) naive and allergic $\mathrm{C} 57 \mathrm{BL} / 6$ mice were analyzed as described in Figure $6 \mathrm{~A}$. Additional details can be found in the Supplemental Methods.

HDM-induced sensitization and restimulation of LN cells. Briefly, $\triangle$ dblGATA or WT control BALB/c mice received an i.n. instillation of saline or $5 \mu \mathrm{g}$ HDM on day O. Five days later, MLN cells were cultured in vitro for 3 days with or without HDM $(30 \mu \mathrm{g} / \mathrm{ml})$. The proliferation was measured as $\left[{ }^{3} \mathrm{H}\right]$ thymidine incorporation during the last 16 hours of a 3-day culture, and culture supernatants were assayed for cytokine production by ELISA (eBioscience). Additional details can be found in the Supplemental Methods.

Coculture experiments and Th2 sensitization induced by i.t. administration of $O V A_{L P S}$-pulsed BMDCs. Methodological details can be found in the Supplemental Methods. 
Statistics. Analyses of data and appropriate statistical tests were performed with the R software package. The different tests used are indicated in the respective figure legends. A $P$ value of less than 0.05 was considered statistically significant.

Study approval. All animal experiments were conducted with the approval of the IACUC of the University of Liège (approval no. 1593).

For immunohistological studies in humans, human lungs were obtained from the International Institute for the Advancement of Medicine (IIAM) (Edison, New Jersey, USA). Ethics approval was obtained from the Royal Brompton \& Harefield Trust (London, United Kingdom; approval no. 09/H0708/72). For flow cytometric studies in humans, human-induced sputa and healthy lung tissue were obtained from the Department of Pneumology and the Department of Thoracic and Cardiovascular Surgery of the CHU of Liège, respectively. Ethics approval was obtained from the Hospital and Medical School Ethics Committee of Liège (approval no. B707201422580). Samples were anonymized, and all participants gave informed consent prior to their participation in the study.

Additional methods are described in the Supplemental Methods.

\section{Author contributions}

FB conceived the project. CM, SR, CD, TM, and FB designed the experiments. FB and TM supervised the project. CM and SR performed most of the experiments and compiled the data. GP and ER performed the immunohistochemical studies and helped with histological and cytological analyses. PS was involved in experiments using phospho-flow cytometry. MAB and MGB provided the human paraffin-embedded samples. MH, FNS, and RL provided sputa from eosinophilic asthmatic patients. MR provided the fresh normal lung tissue. XX, DP, TJ, CD, and LG performed the microarray analyses. KT provided the recombinant GM-CSF and helped with experiments. MT performed TEM experiments and analyzed TEM data. SR, CM, and TM prepared the figures. TM and FB wrote the manuscript, and all authors provided feedback on the manuscript.

\section{Acknowledgments}

We thank James J. Lee (Mayo Foundation for Medical Education and Research, Southwest, Rochester, Minnesota, USA) for providing antiMBP Abs; Natalia Kosovilka from the Stanford PAN Facility; Sandra Ormenese and Raafat Stephan from the Cell Imaging and Flow Cytometry GIGA Platform; Pierre-Vincent Drion, Luc Duwez, Gaëlle Lambert, Laetitia Montero-Ruiz, and other staff members from the Mouse Facility and Transgenics GIGA Platform; Chantal Humblet from the GIGA Immunohistology Platform; Patricia Piscicelli for preparing the samples for electron microscopic analyses; and Ilham Sbai, Raja Fares, and Cédric François for technical and secretarial assistance. The Laboratory of Cellular and Molecular Immunology is supported by grants from the Fonds de la Recherche Scientifique-Fonds National de la Recherche Scientifique (FRS-FNRS) and the Fonds pour la Recherche Fondamentale Stratégique-WELBIO (FRFS-WELBIO) (CR-2012S-01R); the Belgian Program on Interuniversity Attraction Poles (IUAP) T-TIME and P7/30; and an Action de Recherche Concertée de la Fédération Wallonie-Bruxelles de Belgique. SR and TJ are research fellows of the Fonds pour la Formation à la Recherche dans l'Industrie et dans l'Agriculture (FRIA) (FRS-FNRS); SR is supported by the Fonds Léon Frédéricq; PS is supported by a Marie Curie Fellowship of the European Commission (H2020-MSCA-IF-2014, grant number 655153); TM is supported by a Chargé de Recherches postdoctoral fellowship of the FRS-FNRS and by the Acteria Foundation; and CD is a Research Associate of the FRS-FNRS.

Address correspondence to: Fabrice Bureau, Laboratory of Cellular and Molecular Immunology and WELBIO, GIGA Research, B34, University of Liege, Avenue de l'Hôpital 1, 4000 Liège, Belgium. Phone: 32.0.4.366.45.24; E-mail: fabrice.bureau@ulg. ac.be. Or to: Thomas Marichal, Laboratory of Cellular and Molecular Immunology, GIGA-Research, B34, University of Liege, Avenue de l'Hôpital 1, 4000 Liège, Belgium. Phone: 32.0.4.366.95.55; E-mail:t.marichal@ulg.ac.be.
1. Rothenberg ME, Hogan SP. The eosinophil. Annu Rev Immunol. 2006;24:147-174.

2. Travers J, Rothenberg ME. Eosinophils in mucosal immune responses. Mucosal Immunol. 2015;8(3):464-475.

3. Nerlov C, Graf T. PU.1 induces myeloid lineage commitment in multipotent hematopoietic progenitors. Genes Dev. 1998;12(15):2403-2412.

4. Nerlov C, McNagny KM, Döderlein G, Kowenz-Leutz E, Graf T. Distinct C/EBP functions are required for eosinophil lineage commitment and maturation. Genes Dev. 1998;12(15):2413-2423.

5. Hirasawa R, et al. Essential and instructive roles of GATA factors in eosinophil development. J Exp Med. 2002;195(11):1379-1386

6. Bettigole SE, et al. The transcription factor XBP1 is selectively required for eosinophil differentiation. Nat Immunol. 2015;16(8):829-837.

7. Grouls V, Helpap B. Selective staining of eosinophils and their immature precursors in tissue sections and autoradiographs with Congo red. Stain Technol. 1981;56(5):323-325.

8. Rosenberg HF, Dyer KD, Foster PS. Eosinophils: changing perspectives in health and disease. Nat Rev Immunol. 2013;13(1):9-22.
9. Lopez AF, Sanderson CJ, Gamble JR, Campbell HD, Young IG, Vadas MA. Recombinant human interleukin 5 is a selective activator of human eosinophil function. J Exp Med. 1988;167(1):219-224.

10. de Graauw E, Beltraminelli H, Simon HU, Simon D. Eosinophilia in Dermatologic Disorders. Immunol Allergy Clin North Am. 2015;35(3):545-560.

11. Padigel UM, et al. Eosinophils act as antigen-presenting cells to induce immunity to Strongyloides stercoralis in mice. J Infect Dis. 2007;196(12):1844-1851.

12. Shi HZ, Humbles A, Gerard C, Jin Z, Weller PF. Lymph node trafficking and antigen presentation by endobronchial eosinophils. JClin Invest. 2000;105(7):945-953.

13. Wang HB, Ghiran I, Matthaei K, Weller PF. Airway eosinophils: allergic inflammation recruited professional antigen-presenting cells. JImmunol. 2007;179(11):7585-7592.

14. Chu DK, et al. Indigenous enteric eosinophils control DCs to initiate a primary Th2 immune response in vivo. J Exp Med. 2014;211(8):1657-1672.

15. Jacobsen EA, Zellner KR, Colbert D, Lee NA, Lee JJ. Eosinophils regulate dendritic cells and Th2 pulmonary immune responses following allergen provocation. JImmunol. 2011;187(11):6059-6068.

16. Lee JJ, Jacobsen EA, McGarry MP, Schleimer $\mathrm{RP}$, Lee NA. Eosinophils in health and disease: the LIAR hypothesis. Clin Exp Allergy. 2010;40(4):563-575.

17. Chu VT, et al. Eosinophils promote generation and maintenance of immunoglobulin-A-expressing plasma cells and contribute to gut immune homeostasis. Immunity. 2014;40(4):582-593.

18. Jung $Y$, et al. IL-1 $\beta$ in eosinophil-mediated small intestinal homeostasis and IgA production. Mucosal Immunol. 2015;8(4):930-942.

19. Wu D, et al. Eosinophils sustain adipose alternatively activated macrophages associated with glucose homeostasis. Science. 2011;332(6026):243-247.

20. Lumeng CN, Bodzin JL, Saltiel AR. Obesity induces a phenotypic switch in adipose tissue macrophage polarization. JClin Invest. 2007;117(1):175-184.

21. Enerbäck S. Human brown adipose tissue. Cell Metab. 2010;11(4):248-252.

22. Throsby M, Herbelin A, Pléau JM, Dardenne M. CD11c+ eosinophils in the murine thymus: devel- 
opmental regulation and recruitment upon $\mathrm{MHC}$ class I-restricted thymocyte deletion. JImmunol. 2000;165(4):1965-1975.

23. Gouon-Evans V, Pollard JW. Eotaxin is required for eosinophil homing into the stroma of the pubertal and cycling uterus. Endocrinology. 2001;142(10):4515-4521.

24. Gouon-Evans V, Rothenberg ME, Pollard JW. Postnatal mammary gland development requires macrophages and eosinophils. Development. 2000;127(11):2269-2282.

25. Abdala Valencia H, Loffredo LF, Misharin AV, Berdnikovs S. Phenotypic plasticity and targeting of Siglec-F(high) CD11c(low) eosinophils to the airway in a murine model of asthma. Allergy. 2016;71(2):267-271.

26. Carlens J, Wahl B, Ballmaier M, Bulfone-Paus S, Förster R, Pabst O. Common gamma-chaindependent signals confer selective survival of eosinophils in the murine small intestine. JImmunol. 2009;183(9):5600-5607.

27. Rose CE, Lannigan JA, Kim P, Lee JJ, Fu SM, Sung SS. Murine lung eosinophil activation and chemokine production in allergic airway inflammation. Cell Mol Immunol. 2010;7(5):361-374.

28. Dyer KD, Garcia-Crespo KE, Killoran KE, Rosenberg HF. Antigen profiles for the quantitative assessment of eosinophils in mouse tissues by flow cytometry. J Immunol Methods. 2011;369(1-2):91-97.

29. Stevens WW, Kim TS, Pujanauski LM, Hao X, Braciale TJ. Detection and quantitation of eosinophils in the murine respiratory tract by flow cytometry. JImmunol Methods. 2007; 327(1-2):63-74.

30. Takatsu K. Interleukin 5 (IL-5) and its receptor. Microbiol Immunol. 1991;35(8):593-606.

31. Zhang JQ, Biedermann B, Nitschke L, Crocker $\mathrm{PR}$. The murine inhibitory receptor mSiglec-E is expressed broadly on cells of the innate immune system whereas $\mathrm{mSiglec}-\mathrm{F}$ is restricted to eosinophils. Eur JImmunol. 2004;34(4):1175-1184.

32. McGarry MP, Stewart CC. Murine eosinophil granulocytes bind the murine macrophage-monocyte specific monoclonal antibody F4/80. JLeukoc Biol. 1991;50(5):471-478.

33. Gao JL, et al. Identification of a mouse eosinophil receptor for the CC chemokine eotaxin. Biochem Biophys Res Commun. 1996;223(3):679-684.

34. Malm-Erjefält M, Persson CG, Erjefält JS. Degranulation status of airway tissue eosinophils in mouse models of allergic airway inflammation. Am J Respir Cell Mol Biol. 2001;24(3):352-359.

35. Melo RC, Dvorak AM, Weller PF. Contributions of electron microscopy to understand secretion of immune mediators by human eosinophils. Microsc Microanal. 2010;16(6):653-660.

36. Jacobsen EA, Ochkur SI, Lee NA, Lee JJ. Eosinophils and asthma. Curr Allergy Asthma Rep. 2007;7(1):18-26.

37. Wegmann M. Targeting eosinophil biology in asthma therapy. Am J Respir Cell Mol Biol. 2011;45(4):667-674.

38. Marichal T, et al. Interferon response factor 3 is essential for house dust mite-induced airway allergy. J Allergy Clin Immunol. 2010;126(4):836-844.e13.

39. Xue FM, et al. CD98 positive eosinophils con- tribute to T helper 1 pattern inflammation. PLOS ONE. 2012;7(12):e51830.

40. McAlees JW, et al. Distinct Tlr4-expressing cell compartments control neutrophilic and eosinophilic airway inflammation. Mucosal Immunol. 2015;8(4):863-873.

41. Drouin SM, Corry DB, Hollman TJ, Kildsgaard J, Wetsel RA. Absence of the complement anaphylatoxin C3a receptor suppresses Th2 effector functions in a murine model of pulmonary allergy. JImmunol. 2002;169(10):5926-5933.

42. Niebuhr M, Bäumer W, Kietzmann M, Wichmann K, Heratizadeh A, Werfel T. Participation of complement 3 a receptor $(\mathrm{C} 3 \mathrm{aR})$ in the sensitization phase of Th2 mediated allergic contact dermatitis. Exp Dermatol. 2012;21(1):52-56.

43. Mitchell J, Dimov V, Townley RG. IL-13 and the IL-13 receptor as therapeutic targets for asthma and allergic disease. Curr Opin Investig Drugs. 2010;11(5):527-534.

44. Chu DK, et al. Therapeutic potential of anti-IL-6 therapies for granulocytic airway inflammation in asthma. Allergy Asthma Clin Immunol. 2015;11(1):14.

45. Kamal AM, Smith SF, De Silva Wijayasinghe M, Solito E, Corrigan CJ. An annexin 1 (ANXA1)derived peptide inhibits prototype antigen-driven human T cell Th1 and Th2 responses in vitro. Clin Exp Allergy. 2001;31(7):1116-1125.

46. Ng FS, et al. Annexin-1-deficient mice exhibit spontaneous airway hyperresponsiveness and exacerbated allergen-specific antibody responses in a mouse model of asthma. Clin Exp Allergy. 2011;41(12):1793-1803.

47. Oliver PM, et al. Ndfip1 protein promotes the function of itch ubiquitin ligase to prevent $\mathrm{T}$ cell activation and T helper 2 cell-mediated inflammation. Immunity. 2006;25(6):929-940.

48. Fainaru O, et al. Runx3 regulates mouse TGFbeta-mediated dendritic cell function and its absence results in airway inflammation. $Е M B O J$. 2004;23(4):969-979.

49. Benarafa C, Priebe GP, Remold-O’Donnell E. The neutrophil serine protease inhibitor serpinb1 preserves lung defense functions in Pseudomonas aeruginosa infection. JExp Med. 2007;204(8):1901-1909.

50. Yao X, et al. Apolipoprotein E negatively regulates house dust mite-induced asthma via a low-density lipoprotein receptor-mediated pathway. Am J Respir Crit Care Med. 2010;182(10):1228-1238.

51. Collins PD, Marleau S, Griffiths-Johnson DA, Jose PJ, Williams TJ. Cooperation between interleukin-5 and the chemokine eotaxin to induce eosinophil accumulation in vivo. J Exp Med. 1995;182(4):1169-1174.

52. Kopf M, et al. IL-5-deficient mice have a developmental defect in CD5+ B-1 cells and lack eosinophilia but have normal antibody and cytotoxic $\mathrm{T}$ cell responses. Immunity. 1996;4(1):15-24.

53. Furuta GT, Atkins FD, Lee NA, Lee JJ. Changing roles of eosinophils in health and disease. Ann Allergy Asthma Immunol. 2014;113(1):3-8.

54. Yu C, et al. Targeted deletion of a high-affinity GATA-binding site in the GATA-1 promoter leads to selective loss of the eosinophil lineage in vivo. J Exp Med. 2002;195(11):1387-1395.

55. Bedoret $\mathrm{D}$, et al. Lung interstitial macrophages alter dendritic cell functions to prevent airway allergy in mice. JClin Invest. 2009;119(12):3723-3738.

56. DeKruyff RH, Yu S, Kim HY, Umetsu DT. Innate immunity in the lung regulates the development of asthma. Immunol Rev. 2014;260(1):235-248.

57. Hammad H, Lambrecht BN. Barrier Epithelial Cells and the Control of Type 2 Immunity. Immunity. 2015;43(1):29-40.

58. Lambrecht BN, Hammad H. The immunology of asthma. Nat Immunol. 2015;16(1):45-56.

59. Jacobsen EA, et al. Eosinophil activities modulate the immune/inflammatory character of allergic respiratory responses in mice. Allergy. 2014;69(3):315-327.

60. Fattouh R, et al. Eosinophils are dispensable for allergic remodeling and immunity in a model of house dust mite-induced airway disease. Am J Respir Crit Care Med. 2011;183(2):179-188.

61. Barnig C, et al. Circulating Human Eosinophils Share a Similar Transcriptional Profile in Asthma and Other Hypereosinophilic Disorders. PLOS ONE. 2015;10(11):e0141740.

62. Hitoshi Y, et al. Distribution of IL-5 receptor-positive B cells. Expression of IL-5 receptor on Ly-1(CD5)+ B cells. J Immunol. 1990;144(11):4218-4225.

63. Miyajima A, Mui AL, Ogorochi T, Sakamaki K. Receptors for granulocyte-macrophage colony-stimulating factor, interleukin-3, and interleukin-5. Blood. 1993;82(7):1960-1974.

64. Yoshida T, et al. Defective B-1 cell development and impaired immunity against Angiostrongylus cantonensis in IL-5R alpha-deficient mice. Immunity. 1996;4(5):483-494.

65. Håkansson L, Venge P. Priming of eosinophil and neutrophil migratory responses by interleukin 3 and interleukin 5. APMIS. 1994;102(4):308-316.

66. el-Cheikh MC, Borojevic R. Extramedullar proliferation of eosinophil granulocytes in chronic schistosomiasis mansoni is mediated by a factor secreted by inflammatory macrophages. Infect Immun. 1990;58(3):816-821.

67. Lee JJ, et al. Human versus mouse eosinophils: "that which we call an eosinophil, by any other name would stain as red". J Allergy Clin Immunol. 2012;130(3):572-584.

68. Barnes PJ. Immunology of asthma and chronic obstructive pulmonary disease. Nat Rev Immunol. 2008;8(3):183-192.

69. The Jackson Laboratory. Hematological survey of 11 strains of mice. MPD:Jaxpheno4. Mouse Phenome Database web site. The Jackson Laboratory. http://http://phenome.jax.org/db/q?rtn=projects/ projdet\&reqprojid=229. Accessed: June 23, 2016.

70. Riches AC, Sharp JG, Thomas DB, Smith SV. Blood volume determination in the mouse. JPhysiol (Lond). 1973;228(2):279-284.

71. Bentley AM, et al. Identification of T lymphocytes, macrophages, and activated eosinophils in the bronchial mucosa in intrinsic asthma. Relationship to symptoms and bronchial responsiveness. Am Rev Respir Dis. 1992;146(2):500-506.

72. Robinson DS, et al. Predominant TH2-like bronchoalveolar T-lymphocyte population in atopic asthma. N Engl J Med.1992;326(5):298-304.

73. Horikawa K, Takatsu K. Interleukin-5 regulates genes involved in B-cell terminal maturation. Immunology. 2006;118(4):497-508. 
74. Foster PS, Hogan SP, Ramsay AJ, Matthaei KI, Young IG. Interleukin 5 deficiency abolishes eosinophilia, airways hyperreactivity, and lung damage in a mouse asthma model. J Exp Med. 1996;183(1):195-201.

75. Flood-Page PT, Menzies-Gow AN, Kay AB, Robinson DS. Eosinophil's role remains uncertain as anti-interleukin-5 only partially depletes numbers in asthmatic airway. Am J Respir Crit Care Med. 2003;167(2):199-204.

76. Conus S, Straumann A, Bettler E, Simon HU. Mepolizumab does not alter levels of eosinophils, $\mathrm{T}$ cells, and mast cells in the duodenal mucosa in eosinophilic esophagitis. J Allergy Clin Immunol.
2010;126(1):175-177.

77. Kips JC, et al. Effect of SCH55700, a humanized anti-human interleukin- 5 antibody, in severe persistent asthma: a pilot study. Am J Respir Crit Care Med. 2003;167(12):1655-1659.

78. Leckie MJ, et al. Effects of an interleukin- 5 blocking monoclonal antibody on eosinophils, airway hyper-responsiveness, and the late asthmatic response. Lancet. 2000;356(9248):2144-2148.

79. Jacobsen EA, et al. Allergic pulmonary inflammation in mice is dependent on eosinophil-induced recruitment of effector T cells. JExp Med. 2008;205(3):699-710.

80. de Heer HJ, et al. Essential role of lung plasma- cytoid dendritic cells in preventing asthmatic reactions to harmless inhaled antigen. JExp Med. 2004;200(1):89-98.

81. Haley KJ, et al. Immunolocalization of leukotriene $\mathrm{C} 4$ synthase in normal and asthmatic lung. Am J Respir Crit Care Med. 2000;161(3):A926.

82. Kraft M, Martin RJ, Wilson S, Djukanovic R, Holgate ST. Lymphocyte and eosinophil influx into alveolar tissue in nocturnal asthma. Am J Respir Crit Care Med. 1999;159(1):228-234.

83. Willetts L, et al. Immunodetection of occult eosinophils in lung tissue biopsies may help predict survival in acute lung injury. Respir Res. 2011;12:116. 